\title{
Muonium formation by collisions of muons with solid rare-gas and solid nitrogen layers
}

\author{
T. Prokscha, E. Morenzoni, M. Meyberg, ${ }^{*}$ and T. Wutzke ${ }^{\dagger}$ \\ Paul Scherrer Institut, CH-5232 Villigen PSI, Switzerland \\ B. E. Matthias, ${ }^{\ddagger}$ A. Fachat, ${ }^{\S}$ K. Jungmann, and G. zu Putlitz \\ Physikalisches Institut, Universität Heidelberg, D-69120 Heidelberg, Germany
}

(Received 31 March 1998)

\begin{abstract}
We report an observation of the formation of muonium $\left[\mathrm{Mu} \equiv\left(\mu^{+} e^{-}\right)\right.$bound state] with kinetic energies between 1 and $40 \mathrm{keV}$ on 500-nm-thick solid argon, xenon, and nitrogen $\left(\mathrm{N}_{2}\right)$ layers. The thin films are deposited on a $250-\mu \mathrm{m}$-thick aluminum target which is bombarded with a $3.6-\mathrm{MeV} \mu^{+}$beam. We measured the charge state of the muons emerging from the layers as a function of exit energy. The solid layers investigated are of potential interest for the efficient moderation of $\mu^{+}$down to energies of $10 \mathrm{eV}$ for use as a source for a low-energy $\mu^{+}$beam. For the cryogenic insulators investigated, we find that the measured energydependent neutral fraction of the exiting muon beam is well interpreted by velocity scaling of known cross sections of protons for electron capture and electron loss down to muon energies of $1 \mathrm{keV}$. The experimental results are well reproduced by a Monte Carlo simulation. The total fraction of muonium in the exiting beam is found to be $2 \times 10^{-3}$ for argon and nitrogen as well as for the aluminum substrate without deposited layer, and about $20 \%$ less than this for xenon. [S1050-2947(98)07411-3]
\end{abstract}

PACS number(s): 34.70.+e, 36.10.Dr, 34.50.Bw, 79.90.+b

\section{INTRODUCTION}

Besides excitation and ionization, charge-exchange reactions like electron capture and electron loss are fundamental processes in understanding ion-atom collisions. At energies below a few tens of $\mathrm{keV} /$ nucleon these are the dominant inelastic processes. Over the last 40 years there has been extensive study of charge-changing processes for protons, deuterons, and helium ions of kinetic energy below $1 \mathrm{MeV} /$ nucleon passing through gases or thin foils [1-6]. These experimental and theoretical studies show that the cross sections for charge exchange depend on the velocity of the projectile but not on its mass. This is known as the velocity scaling of cross sections. For example, for deuterons with energies between 25 and $170 \mathrm{keV} /$ nucleon the measured charge fractions $\mathrm{D}^{+}, \mathrm{D}^{0}$ and $\mathrm{D}^{-}$on several materials are the same as for protons with equal velocity [5]. Also, for heavier charged ions $(Z>1)$ extensive data on equilibrium charge fractions are available [7]. For instance, in charge-changing collisions of the neon isotopes ${ }^{20} \mathrm{Ne}$ and ${ }^{22} \mathrm{Ne}$ with thin carbon foils, it was shown that the neutral fractions of both isotopes are the same at equal velocity in the energy range of $0.5-2.0 \mathrm{keV} /$ nucleon [8]. However, the comparison between proton data and heavier charged ion data or between different heavier ion data is complicated by the fact that in a heavy ion the complex many-electron structure leads to a large variety of excitations and many-electron processes which

\footnotetext{
*Present address: DMP AG, Ch-8604 Volketswil, Switzerland.

${ }^{\dagger}$ Present address: Petristrasse 6, D-38118 Braunschweig, Germany.

${ }^{\ddagger}$ Present address: ABB Research Corporation, D-69115 Heidelberg, Germany.

${ }^{\S}$ Present address: Institut für Physik, Technische Universität Chemnitz, D-09107 Chemnitz, Germany.
}

somewhat obscure the details of the charge-changing reaction.

An ideal projectile for studying velocity scaling is the positively charged muon $\left(\mu^{+}\right)$which has a mass of about $1 / 9$ proton masses $\left(m_{\mu}=m_{p} / 8.88\right)$. It decays into a positron and two neutrinos $\left(\mu^{+} \rightarrow e^{+} \overline{\nu_{\mu}} \nu_{e}\right)$ with a lifetime of $\tau_{\mu}$ $=2.197 \mu \mathrm{s}$ [9]. In atomic collisions it may be considered as a light proton isotope with its neutral and negatively charged counterparts muonium $\mathrm{Mu}\left[\equiv\left(\mu^{+} e^{-}\right)\right.$the hydrogenlike bound state $]$ and $\mathrm{Mu}^{-}\left[\equiv\left(\mu^{+} e^{-} e^{-}\right)\right]$, respectively. So far, however, only few data on muon-atom interactions are available. Since energy ranges and fluxes for $\mu^{+}$beams are quite limited the experiments are performed mainly with relatively thick $(\sim \mu \mathrm{m})$ targets. Data obtained from experiments with metal and carbon foils [10-13] indicate the validity of velocity scaling for atomic interactions of $\mu^{+}$. These include charge exchange, ionization, and excitation processes.

Detailed knowledge of the charge-changing processes of $\mu^{+}$would be also very useful for the efficient formation in vacuum of the $\mathrm{Mu}$ atom or of the negatively charged $\mathrm{Mu}^{-}$ ion. These hydrogenlike systems are ideal for spectroscopic tests of the validity of quantum electrodynamics (QED), since the leptonic constituents of $\mathrm{Mu}$ and $\mathrm{Mu}^{-}$behave as pointlike particles down to dimensions of less than $10^{-18} \mathrm{~m}$ [14]. Numerous spectroscopic experiments on Mu have been performed or are currently underway concerning the $1 s-2 s$ interval, the hyperfine splitting in the ground state, the $2 s_{1 / 2}-2 p_{1 / 2}$ Lamb shift, and the $2 s_{1 / 2}-2 p_{3 / 2}$ fine-structure splitting [15]. Besides spectroscopic experiments, the conservation of lepton number in electroweak interactions is probed by searching for muonium to antimuonium $\left(\mu^{-} e^{+}\right)$ oscillations [16].

An improved understanding of the charge-changing processes of a $\mu^{+}$interacting with matter is also relevant for the application of polarized $\mu^{+}$as a magnetic microprobe in 
condensed-matter studies ( $\mu \mathrm{SR}$ techniques [17]). In these investigations, polarized $\mu^{+}$with energies of a few $\mathrm{MeV}$ are implanted in matter, where they slow down and thermalize as positive muons or as neutral muonium. The information about the muon spin at the moment of the decay, and hence about local fields, field distributions, magnetic phase transitions, and muon diffusion, is obtained by measuring the time-dependent angular distribution of the positron originating from the anisotropic decay of the $\mu^{+}$. In gases the final charge-state fractions of the $\mu^{+}$are essentially determined by charge-changing cycles involving electron capture (muonium formation) and electron loss (muonium breakup) $[18,19]$. The last charge-changing cycles take place at energies above a few eV. The muonium formed at these epithermal energies will thermalize by elastic collisions and determine the observed fraction of stopped muonium. The remaining $\mu^{+}$below a few eV no longer form muonium. In solids these cycles also play an important though perhaps not such a central role. The thermalized $\mu^{+}$can interact with the spur electrons generated during the slowing down of the $\mu^{+}$ by ionization if the mobility of these electrons is sufficiently high. Therefore, in solids, the formation of thermal muonium is influenced by the possible convergence of the stopped $\mu^{+}$ with a spur electron. This was recently shown for solid $\mathrm{N}_{2}$ [20], where the formation of thermal muonium was found to depend partly on the applied electric field. The fraction of thermalized muonium is increased by a field which pushes the $\mu^{+}$and the electrons together, and it is decreased with the opposite field direction. Also, the observation of muonium in superfluid liquid helium $\left({ }^{4} \mathrm{He}\right)[21]$ and in liquid and solid neon [22] is explained by the convergence of thermalized $\mu^{+}$with a spur electron.

The same charge-changing cycles contribute significantly to the moderation of fast $\mu^{+}$, which is particularly relevant for the generation of a beam of very slow, polarized $\mu^{+}$with energies of a few eV. To date, the most efficient method to produce such a beam appears to be a moderation technique, where fast $\mu^{+}$with energies of a few MeV slow down in a moderator formed by condensation of a van der Waals solid layer on a metal substrate [23-26]. The most suitable layers are currently made of rare-gas solids (RGS's) or of solid nitrogen $\left(\mathrm{N}_{2}\right)$. The studies presented here are also motivated by our development of a low-energy $\mu^{+}$beam [25]. The usual interpretation of the slowing down in the moderator distinguishes a high-, a medium- and a low-energy regime. At high energies, where the velocity $v_{\mu}$ of the $\mu^{+}$is much greater than the velocity $v_{e}$ of the atomic electrons, energy loss is caused by ionization, and is well described by the Bethe-Bloch formula [9]. At medium energies, where $v_{\mu}$ $\sim v_{e}$ corresponding to $\mu^{+}$energies of about $3 \mathrm{keV}$, energy loss is dominated by charge-changing cycles. At energies below a few eV, in gases only elastic collisions contribute to the energy loss, whereas in solids processes like phonon excitations in principle are also possible. So far, however, there is no detailed understanding of the moderation of $\mu^{+}$in RGS's or $\mathrm{N}_{2}$ layers at low energies; in particular, there is a lack of knowledge regarding the role played by $\mathrm{Mu}$ formation at low energies.

We have performed studies on the formation of $\mathrm{Mu}$ atoms with kinetic energies of a few $\mathrm{keV}$ emerging from solid argon $(\mathrm{Ar})$, xenon $(\mathrm{Xe})$, and $\mathrm{N}_{2}$ layers and also from the metal substrate (aluminum, Al) without a frozen gas layer. Due to their simplicity and ready availability, collisions of hydrogen atoms and ions with rare gases and gaseous $\mathrm{N}_{2}$ have been studied extensively. Energy-dependent cross sections for electron capture and electron loss have been summarized and described by fitting empirical formulas to the measured cross sections in Ref. [27]. These cross sections determine the charge fractions in the exiting beam. By comparing the scaled charge fractions with our experimental data, a more detailed understanding of the atomic interaction of the $\mu^{+}$ can be obtained than is possible from metal or carbon foil experiments.

In Sec. II the experimental setup and the data acquisition are described in detail. In Sec. III the data analysis is presented, and the experimental neutral fraction is compared with the calculated result obtained by velocity scaling of the cross sections for charge transfer of protons. In Sec. IV the influence of solid-state effects on our result is discussed, and also the consistency of our data with the velocity scaled results down to $\mu^{+}$energies of $1 \mathrm{keV}$. Additionally, the validity of velocity scaling at $\mu^{+}$energies below $1 \mathrm{keV}$ is considered with its consequences on the $\mu^{+}$moderation at low energies. Finally, the results are summarized in Sec. V.

\section{EXPERIMENTAL DETAILS}

The experiment was performed at the Paul Scherrer Institute (PSI, Villigen, Switzerland), where a 50-MHz cyclotron delivers protons with an energy of $590 \mathrm{MeV}$ to produce intense secondary beams of charged pions and muons by traversing two graphite targets. The muons originate from charged pion decays and form continuous muon beams. This experiment used the $\pi E 1$ beamline, which was tuned to deliver $\mu^{+}$with a momentum of $27.5 \mathrm{MeV} / c$, corresponding to a kinetic energy of $3.6 \mathrm{MeV}$, and a momentum width $\Delta p / p$ of $2 \%$ full width at half-maximum (FWHM), corresponding to an energy width of $0.14 \mathrm{MeV}$. The beam is transported and focused using standard magnetic quadrupoles and dipoles. A static separator with a crossed electric and magnetic field $(\mathbf{E} \times \mathbf{B})$ reduces an $e^{+}$contamination originating from neutral pion decays to the level of a few percent. Radioactive gases, generated in nuclear reactions of the protons in the graphite target and diffusing through the beamline, are stopped in a gas barrier consisting of a 2.0- $\mu \mathrm{m}$ Mylar window at the end of the beamline. Behind the gas barrier our apparatus, shown in Fig. 1, is connected with the beamline. It is a modified version of the apparatus used for our studies of the $\mu^{+}$moderation technique [25]. Its main component consists of an UHV chamber with a base pressure of $10^{-10}$ mbar. Inside the chamber the $\mu^{+}$impinge on a cryogenic target consisting of an $\mathrm{Al}$ foil with a thin gas layer deposited on the downstream side. In charge-exchange collisions with the atoms of the layer, equilibrium fractions of $\mu^{+}$and $\mathrm{Mu}$ are formed and exit the layer. The dominant fraction of the exiting muons remains positively charged. With a bending magnet on the downstream side of the target the charged particles of the exiting beam can be separated from the neutral ones. Time-of-flight (TOF) spectra are measured for the two cases, magnet switched on and off, corresponding to the TOF spectra of $\mathrm{Mu}$ and all muon charge states $\left(\mu^{+}, \mathrm{Mu}, \mathrm{Mu}^{-}\right)$, respectively. The TOF spectra are 


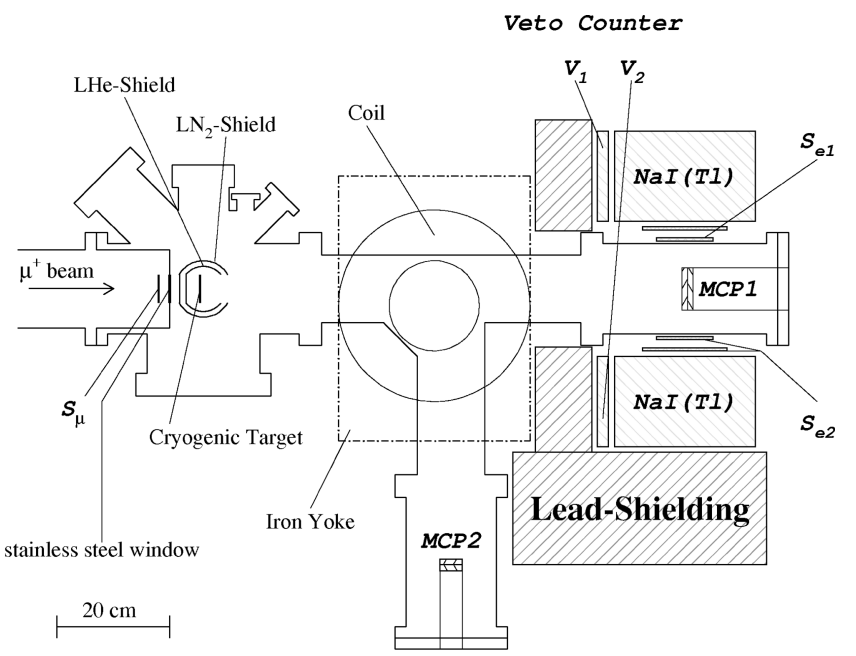

FIG. 1. Schematic of the experimental setup; see text for details. The magnetic field inside the coil is directed perpendicularly upwards from the plane of view.

converted into energy spectra to calculate the energydependent neutral fraction defined as the ratio of the $\mathrm{Mu}$ energy spectrum to the energy spectrum of all muon charge states. In the following we give a more detailed description of the experiment.

\section{A. Incident beam and cryogenic target}

The incident $\mu^{+}$are detected after collimation when passing through a $200-\mu \mathrm{m}$-thick plastic scintillator $\left(S_{\mu}\right.$, NE102A material, Nuclear Enterprises) which gives the start signal for the TOF measurement. The $\mu^{+}$enter the UHV chamber through a $50-\mu \mathrm{m}$ stainless-steel window, which separates the UHV conditions in the chamber from the lesser vacuum of the order of $10^{-6} \mathrm{mbar}$ in the beamline.

Inside the apparatus, the $\mu^{+}$traverse two cooled, $30-\mu \mathrm{m}$-thick, $\mathrm{Al}$ windows before they impinge on the central component of the chamber, the cryogenic target. It is composed of a $250-\mu \mathrm{m}$-thick Al foil of $99.999 \%$ purity, cooled by a liquid-helium flow cryostat with temperature control and either covered on the downstream side with a $\sim 500$-nm thin solid layer of Ar, $\mathrm{Xe}$, or $\mathrm{N}_{2}$, or left uncovered (Al). The film thickness of $500 \mathrm{~nm}$ is sufficient to obtain an equilibrium of charge states inside the layers [28]. The Al substrate is mounted between two gold-plated frames of copper with a total thickness of $5 \mathrm{~mm}$. The target is surrounded by two shields of gold plated, oxygen-free high-conductivity copper (OFHC, each $1.5 \mathrm{~mm}$ thick) to reduce the exposure of the films to thermal radiation. The outer shield is cooled by a liquid nitrogen reservoir, and the inner one by the liquid helium reservoir. To avoid the absorption of the incoming $\mu^{+}$the two Al windows are mounted in the shields on the upstream side. On the downstream side an opening is left for the particles emerging from the target region.

The base temperature at the target substrate is measured to be $10 \mathrm{~K}$. The temperature is controlled with LakeShore Si diodes, and stabilized within $0.1 \mathrm{~K}$. The thin-film layer is formed by condensing research purity gas on the cold Al substrate, where the temperature for the $\mathrm{Ar}$ and $\mathrm{N}_{2}$ layers is set to $15 \mathrm{~K}$, and that for the Xe layer to $35 \mathrm{~K}$. The gas, regulated with a high-precision leak valve, is admitted into the vacuum chamber through a directional gas doser, mounted on a manipulator which is positioned $10 \mathrm{~cm}$ downstream of the substrate during the deposition procedure. The film grows at a partial pressure of $2 \times 10^{-5}$ mbar for about 2 min. After termination of the deposition procedure, the residual pressure returns to the $10^{-10}$-mbar range within a few minutes. The gas composition in the UHV chamber is monitored with a quadrupole mass spectrometer. The substrate is heated up to $120 \mathrm{~K}$ every $8 \mathrm{~h}$ to remove the old layer, and a new one is prepared after cooling back down. This procedure and the UHV conditions in the chamber ensure the cleanness of the film surface over a measurement, so that no influence from residual gas contaminants on the Mu formation is expected. The thickness of the film is measured with a quartz microbalance (standard frequency shift method) placed adjacent to the substrate and also cooled by the cryostat.

\section{B. Beam-target interaction and time-of-flight measurement}

The beam momentum of $27.5 \mathrm{MeV} / c$ is determined by our $\mu^{+}$moderation studies, where an intense, highly polarized $\mu^{+}$beam is required. A $\mu^{+}$polarization of nearly $100 \%$ is obtained with the so-called surface and subsurface $\mu^{+}$ beams with momenta $\leqslant 29.8 \mathrm{MeV} / c$ [29]. With a beam momentum of $27.5 \mathrm{MeV} / c$ and an amount of material of about $150 \mathrm{mg} / \mathrm{cm}^{2}$ traversed by the $\mu^{+}$, the stopping density distribution is centered at the downstream side of the target, i.e., in the cryogenic solid layers of condensed gas. This maximizes the rate of $\mu^{+}$with energies of a few $\mathrm{keV}$ coming out of the solid layers, and also the integral rate of $\mathrm{Mu}$ atoms. About $90 \%$ of the beam stops in the Al substrate, or in the copper frame due to multiple scattering in the material in front of the target (see Sec. III F). The remaining fraction of $10 \%$ of the beam emerges from the target. A small part of this fraction hits a microchannel plate detector (MCP1) producing the stop signal of the TOF measurement. The largest part of the exiting beam misses the MCP1 detector or stops in the vacuum tube in front of the MCP1 due to the multiple scattering of the beam in the target region. The exiting $\mu^{+}$ have lost most of the initial energy and the energy distribution is significantly broadened. The mean energy amounts to approximately $500 \mathrm{keV}$, with a FWHM of the same order of magnitude. The time zero $t_{\mu}$ of the TOF measurement, defined as the time when the $\mu^{+}$or Mu leave the target, is obtained from the TOF of the contaminating beam $e^{+}$after the separator. At a momentum of $27.5 \mathrm{MeV} / c$ the $e^{+}$have a velocity $v \approx c$, and appear as a sharp peak in the TOF spectrum. From the peak position the time $t_{S_{\mu}}$ when particles are passing the start scintillator $S_{\mu}$ can be calculated using the known distance between $S_{\mu}$ and MCP1. The time zero $t_{\mu}$ is calculated by adding to $t_{S_{\mu}}$ the time of flight of the $\mu^{+}$from $S_{\mu}$ to the target $(1.6 \mathrm{~ns})$.

\section{Separation of charged and neutral beam components}

A bending magnet with circular coils, contained in an iron yoke, is located between the target and the MCP1 detector. When the magnet is switched on ( $B$ field on), the charged particles are deflected into the side branch of the apparatus and monitored by a second microchannel plate detector 
(MCP2) which is mounted $20 \mathrm{~cm}$ behind the focal plane of the magnet. The magnet is set to a field of $0.43 \mathrm{~T}$ to deflect muons with a momentum of $13 \mathrm{MeV} / c$ by $90^{\circ}$, corresponding to a muon energy of $800 \mathrm{keV}$. The best setting of the $B$ field is found by maximizing the MCP2 rate as a function of the field strength. With the resulting magnet setting no $\mu^{+}$are detected at MCP1.

\section{Detection of $e^{+}$from $\mu^{+}$decays}

For background reduction, especially for the Mu TOF measurement, a coincidence between MCP1 and the delayed signal of two pairs of plastic scintillator paddles $S_{e i}[\mathrm{i}=$ $(1,2)]$ surrounding MCP1 is used, where the $S_{e i}$ detect the $e^{+}$ originating from the $\mu^{+}$decay in MCP1. Each scintillator has a thickness of $4 \mathrm{~mm}$, and is made of NE102A material. The lifetime spectrum of the $\mu^{+}$is obtained by measuring the time difference between the signals of MCP1 and one of the $S_{e i}$ detectors. Additionally, the characteristic energy spectrum of the decay $e^{+}$[30] passing the $S_{e i}$ detectors is measured with $\mathrm{NaI}(\mathrm{Tl})$ crystals. Two veto counters $\left(V_{1,2}\right.$, 2-cm-thick plastic scintillators, NE102A material) allow the rejection of events due to $e^{+}$originating from $\mu^{+}$decays in the side arm of the apparatus. These events are strongly suppressed by a $20-\mathrm{cm}$-thick lead shield between the iron yoke of the bending magnet and the rear part of the apparatus with the MCP1, $S_{e i}$ and $\mathrm{NaI}(\mathrm{Tl})$ detectors.

\section{E. Detector efficiencies}

The MCP1 detector has a circular active area of $75 \mathrm{~mm}$ in diameter. It is located at a distance of $86.4(3) \mathrm{cm}$ from the target subtending a solid angle of $\Omega_{\mathrm{MCP} 1}=9.5(5) \times 10^{-4}$ of $2 \pi$ sr. However, since the muons do not exit the target isotropically but are forward distributed, the acceptance of MCP1 with respect to the $\mu^{+}$or Mu angular distribution is larger than $\Omega_{\mathrm{MCP} 1}$. This is discussed in detail in Sec. III F. The detection efficiency $\epsilon_{\mathrm{MCP} 1}$ of MCP1 for $\mu^{+}$and Mu is assumed to be about $50 \%$, and approximately constant over the energy range between $1 \mathrm{keV}$ and $1 \mathrm{MeV}$ [31]. The uniformity of $\epsilon_{\mathrm{MCP} 1}$ over the energy range of the exiting $\mu^{+}$is an important detail for the data analysis. The detection efficiency $\epsilon_{e+}$ for decay $e^{+}$of each $S_{e i}$ detector has been determined by comparing the number of $\mu^{+}$detected in the TOF spectrum to the number of decay $e^{+}$detected in the $\mu^{+}$ lifetime spectrum. We obtain $\epsilon_{e+}=5.7(8) \%$. This is consistent with the result from a Monte Carlo simulation using the program GEANT [32], which takes into account the interaction of the $e^{+}$traversing MCP1 and the material surrounding MCP1. The simulation shows that only $64 \%$ of the $e^{+}$originating from $\mu^{+}$decays in MCP1 can be detected in the $S_{e i}$. The losses are due to scattering and absorption in MCP1 and the vacuum tube. Together with the solid angle of $10.0(0.5) \%$ of each scintillator pair subtended at MCP1 and the detection efficiency of approximately $90 \%$ for the decay positrons in each pair, the simulation agrees with the measured value of $\epsilon_{e+}$.

\section{F. Trigger logic and data acquisition}

The data are recorded event by event. The acquisition of a valid event is triggered by a triple coincidence of $S_{\mu}$, MCP1,

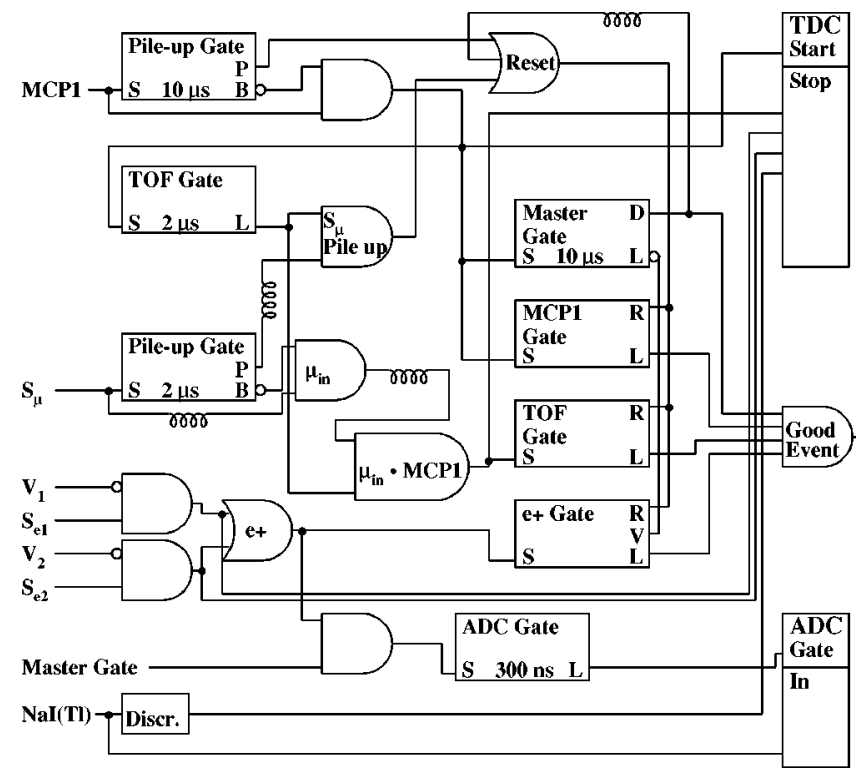

FIG. 2. Schematic of the trigger electronics. All input signals are NIM logic pulses except for the $\mathrm{NaI}(\mathrm{Tl})$ signal, which is the analog pulse. Only one of the $\mathrm{NaI}(\mathrm{Tl})$ signals is shown here. $S$ : start; $L$ : latched; $B$ : busy; $P$ : pile-up; $D$ : delayed; $R$ : reset; $V$ : veto.

and one of the scintillator pairs using standard NIM electronics. For the $\epsilon_{e+}$ measurement only a coincidence between $S_{\mu}$ and MCP1 is required. A schematic of the electronics is shown in Fig. 2. A MCP1 pulse must occur within $2 \mu$ s after a $S_{\mu}$ pulse has been detected, and a delayed decay $e^{+}$is required to be detected in one of the scintillator pairs within $10 \mu \mathrm{s}$ after the MCP1 signal. To ensure the correlation between $S_{\mu}$ and MCP1 signals, only those $S_{\mu}$ hits are accepted for the TOF coincidence which are not preceeded by a second $S_{\mu}$ for at least $2 \mu \mathrm{s}$. The corresponding signal is $\mu_{\text {in }}$. Additionally, an event is rejected if a $\mu_{\text {in }}$ is followed by a second $S_{\mu}$ during the TOF gate. The MCP1 signal is required to be not preceeded or followed by a second hit for the duration of $10 \mu \mathrm{s}$. This ensures the clean correlation between MCP1 and $S_{e i}$ signals. An additional pile-up rejection for the $S_{e i}$ detectors is not necessary due to the low maximum detection rates of a few hundred counts/s. The accepted beam rate $\mu_{\mathrm{in}}^{\text {acc }}$ is reconstructed from single detector and coincidence rates. It denotes the remaining fraction of the $\mu_{\text {in }}$ rate after pile-up rejection, and is needed for normalization and for background rate estimates. The MCP1 signal is used to start both the TOF and $\mu^{+}$lifetime measurements to record the spectra simultaneously with only one start signal. Therefore, the $\mu_{\text {in }}$ signal is delayed by $1.8 \mu$ s to stop the TOF measurement, so that the TOF spectra are recorded with reverse timing. In the data analysis (Sec. III) the TOF spectra are shown with forward timing. The pulses of the $\mathrm{NaI}(\mathrm{Tl})$ detectors are charge integrated to measure the energy deposited by the $e^{+}$. Additionally, the signals are discriminated using constant fraction discriminators to use them also to provide the stop for the lifetime measurement. The time spectra are digitized using a LeCroy TDC4208 in single hit mode. The pulse heights of the $\mathrm{NaI}(\mathrm{Tl})$ crystals are recorded using a charge integrating LeCroy ADC 2249W. Single detector and coincidence rates are monitored with CAMAC scalers. The CAMAC modules are controlled and read out by 
a CES Starburst ACC 2180. The data are sent to a VAXstation 4000-90, where they are stored on disk and analyzed. The slowly varying parameters such as target temperature, pressure, residual gas composition, and film thickness are monitored and sent to the VAXstation every few minutes, where they are also stored on disk.

\section{DATA ANALYSIS AND RESULTS}

\section{A. General scheme of the analysis}

The objective of the analysis is to extract the neutral fraction of the secondary muon beam downstream of the cryogenic target and to compare the experimental result with the calculated neutral fraction using known, velocity scaled cross sections of protons for electron capture $\sigma_{c}$ and electron loss $\sigma_{l}$ for hydrogen.

The neutral fraction $\Phi_{0}(E)$ as a function of muon energy $E$ is obtained by converting the Mu TOF spectra $N_{\mathrm{Mu}}(t) \quad(B$ field on) and the TOF spectra $N_{\text {tot }}(t)$ ( $B$ field off) to the corresponding energy spectra $N_{\mathrm{Mu}}(E)$ and $N_{\text {tot }}(E)$, and forming the ratio

$$
\Phi_{0}(E)=\frac{N_{\mathrm{Mu}}(E)}{N_{\mathrm{tot}}(E)} .
$$

The energy spectra are properly normalized to the accepted number of incoming $\mu^{+}\left(\mu_{\mathrm{in}}^{\mathrm{acc}}\right)$. They are extracted from the TOF spectra using the general relation

$$
N(E)=N(t)\left(\frac{d E}{d t}\right)^{-1}
$$

where

$$
\begin{gathered}
\frac{d E}{d t}=-\gamma^{3} m l^{2} t^{-3}, \\
\gamma=\left[\sqrt{1-(l / c t)^{2}}\right]^{-1},
\end{gathered}
$$

in which $m$ denotes the mass of the particle, $l$ is the distance between target and MCP1, and $c$ is the velocity of light. The experimental data and also a simulation, described below, show that the energy spectrum $N_{\text {tot }}(E)$ is a Gaussian to good approximation. In this case, the TOF spectrum $N_{\text {tot }}\left(t^{i}\right)$ at bin $i$ can be written as (see Appendix A)

$$
N_{\text {tot }}\left(t^{i}\right)=\frac{N_{\text {tot }}^{\text {sum }}}{\operatorname{erfc}\left(-\frac{E_{\mu}}{\sqrt{2} \sigma_{\mu}}\right)}\left[\operatorname{erf}\left(x_{l}^{i}\right)-\operatorname{erf}\left(x_{h}^{i}\right)\right]+B\left(t_{h}^{i}-t_{l}^{i}\right),
$$

where $E_{\mu}$ and $\sigma_{\mu}$ are the mean energy and the standard deviation of the Gaussian distribution, $N_{\text {tot }}^{\text {sum }}$ is the total number of muons in the TOF peak, $B$ is a flat background contribution per channel, which is a good approximation in the vicinity of the $\mu^{+}$peak, and $t_{h}^{i}$ and $t_{l}^{i}$ are the upper and lower boundary of time bin $i$. The arguments $x^{i}$ of the error function erf are given in detail in Appendix A, and erfc denotes the complementary error function.
Knowing the energy-dependent cross sections $\sigma_{c}(E)$ and $\sigma_{l}(E)$ the neutral fraction can be written as [2]

$$
\Phi_{0}(E)=\frac{\sigma_{c}(E)}{\sigma_{c}(E)+\sigma_{l}(E)} .
$$

The cross sections $\sigma_{c}(E)$ and $\sigma_{l}(E)$ are measured for protons in various gases. For $\mathrm{Ar}, \mathrm{Xe}$, and $\mathrm{N}_{2}$, measurements were done down to energies of $\sim 100 \mathrm{eV}$. For the calculation of $\Phi_{0}(E)$, we use the analytical formulas presented in the compilation of Ref. [27]. The velocity-scaled cross sections for $\mu^{+}$at energy $E$ are given by $\sigma_{c, l}(E)=\sigma_{c, l}\left(E_{p} m_{\mu} / m_{p}\right)$ with the proton energy $E_{p}$. In Eq. (4) the formation of the negative $\mathrm{Mu}^{-}$ion is neglected since the formation cross sections are at least one order of magnitude smaller than $\sigma_{c}(E)$ and $\sigma_{l}(E)$.

We define the integral neutral fraction $\Phi_{0}^{\text {sum }}$ by forming the ratio of the total number $N_{\mathrm{Mu}}^{\text {sum }}$ of Mu with the total number $N_{\text {tot }}^{\text {sum }}$ of $\mu^{+}$in the beam downstream of the target:

$$
\begin{aligned}
\Phi_{0}^{\mathrm{sum}} & =\frac{N_{\mathrm{Mu}}^{\mathrm{sum}}}{N_{\mathrm{tot}}^{\mathrm{sum}}}=\frac{\int N_{\mathrm{Mu}}(E) d E}{\int N_{\mathrm{tot}}(E) d E} \\
& =\frac{\int \frac{\sigma_{c}(E)}{\sigma_{c}(E)+\sigma_{l}(E)} N_{\mathrm{tot}}(E) d E}{\int N_{\mathrm{tot}}(E) d E} .
\end{aligned}
$$

Experimentally, we determine the second term of Eq. (5), $N_{\mathrm{Mu}, \mathrm{MCP} 1}^{\mathrm{sum}} / N_{\mathrm{tot}, \mathrm{MCP} 1}^{\mathrm{sum}}$, where the subscript MCP1 means the detected fraction on the MCP1 detector. This number is compared with the last term of Eq. (5), where again we use the scaled cross sections together with a simulated energy spectrum $N_{\text {tot,MCP1 }}(E)$. The simulated energy spectrum is obtained by calculating the energy loss, energy straggling, and multiple scattering of the $\mu^{+}$in the target using velocity scaled proton data. The simulation will show that the energy spectrum of the total exiting beam is different from that fraction of the beam hitting MCP1. Therefore, the integral fraction $\Phi_{0}^{\text {sum }}$ of the total beam will differ from $\Phi_{0, \mathrm{MCP} 1}^{\text {sum }}$ with $\Phi_{0}^{\text {sum }}>\Phi_{0, \mathrm{MCP} 1}^{\mathrm{sum}}$.

\section{B. Analysis of the $\boldsymbol{\mu}^{+}$and Mu time-of-flight data}

The description of the analysis procedure is confined to the example of one Ar run. The measured time-of-flight spectra $N_{\text {tot }}(t)$ and $N_{\mathrm{Mu}}(t)$ are shown in Figs. 3 and 4, respectively. Cuts, described later in Sec. III C, are applied in the analysis to reduce the background. This is essential for the extraction of the Mu TOF spectra.

Figure 3(a) shows the $N_{\text {tot }}(t)$ spectrum with a fit using the function of Eq. (3). In Fig. 3(b), the same spectrum is converted first into an energy spectrum using Eq. (2), and subsequently, a Gaussian is fitted to $N_{\text {tot }}(E)$. Both methods give, within the errors, the same mean energy and standard deviation. The knowledge that the energy distribution is a Gaussian is useful in order to reduce the statistical error per bin in the $N_{\text {tot }}(E)$ spectrum. The fit parameters of the Gaussian 

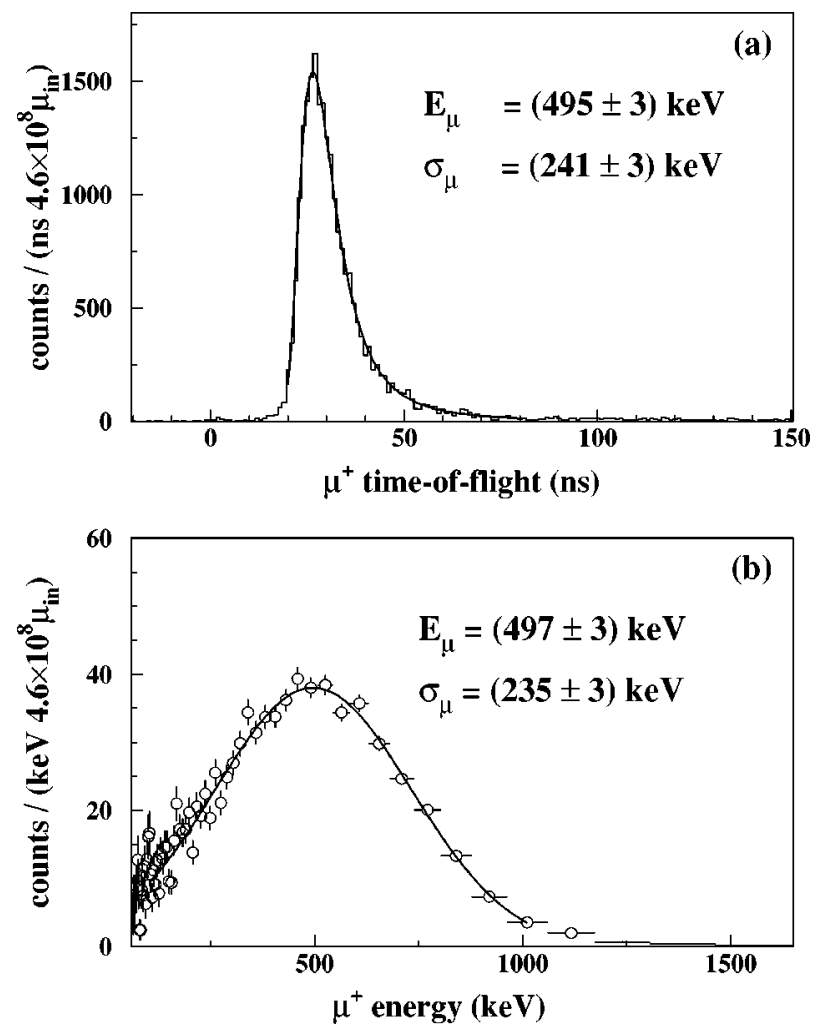

FIG. 3. (a) Measured time-of-flight spectrum for $\mu^{+}$with software cuts applied (see Sec. III C), fitted with the function given in Eq. (3). (b) The TOF spectrum is first converted into an energy spectrum, where subsequently a Gaussian is fitted. The horizontal error bars are showing the variable bin size in the energy spectrum.

$\left(N_{\text {tot }}^{\text {sum }}, E_{\mu}, \sigma_{\mu}\right)$ are extracted from the fit with small errors. The fitted energy spectrum shows no statistical fluctuations and the errors per bin are calculated by standard error propagation using the errors of the fit parameters. Therefore, the errors per bin in the fitted spectrum can be regarded as an average of the statistical fluctuations over the total $N_{\text {tot }}(E)$ spectrum. The error per bin in the fitted spectrum, especially at low energies where $\mathrm{Mu}$ formation is expected, is smaller than the errors in the $N_{\text {tot }}(E)$ spectrum, where the error per bin $i$ is given by $\sqrt{n_{i}}$ with $n_{i}$ the number of counts in the corresponding time bin in the TOF spectrum. For the calculation of the neutral fraction, in Eq. (1) we use the $N_{\text {tot }}(E)$ spectrum obtained from the fit to the TOF spectrum.

The raw Mu spectrum in Fig. 4(a) shows no structure apart from a prompt peak at $t \approx 0$ due to scattered beam $e^{+}$ and a decreasing background. The fitted background between 0.1 and $1.6 \mu \mathrm{s}$ is discussed below and in Appendix B. Only after applying cuts does the broad Mu TOF distribution between 0.1 and $1 \mu \mathrm{s}$ become visible, corresponding to $\mathrm{Mu}$ energies of 40 and $0.4 \mathrm{keV}$, respectively [see Fig. 4(b)]. We use Eq. (2) to determine the energy spectrum since there is no theoretical model to predict the shape of the energy spectrum. The background in Fig. 4(b) is indicated as a dashed line and is discussed below. To give an example of the importance of the correct background subtraction to obtain the $\mathrm{Mu}$ energy spectrum at low energies, one should notice that, after background subtraction, the total number of detected $\mathrm{Mu}$ atoms amounts to $N_{\mathrm{Mu}}^{\mathrm{sum}}=50(9)$, yielding a detected $\mathrm{Mu}$ rate of $0.004 / \mathrm{s}$ at an accepted beam rate of $\mu_{\text {in }}^{\text {acc }}=10^{5} / \mathrm{s}$. The
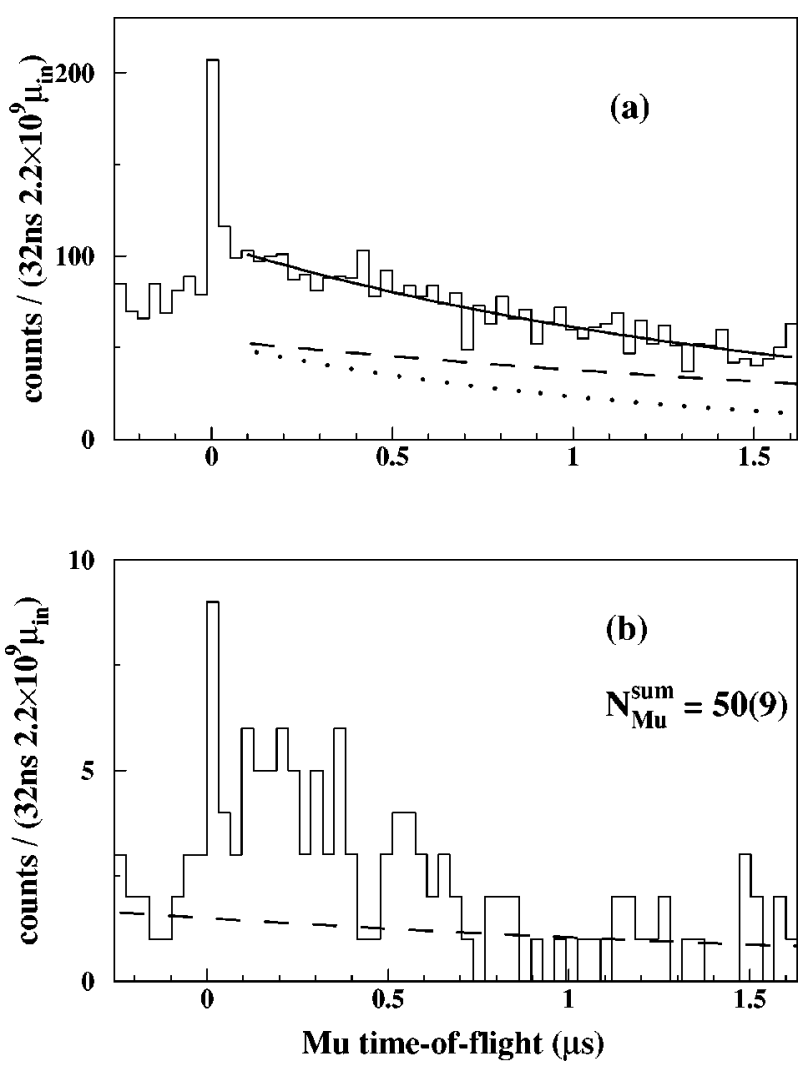

FIG. 4. Time-of-flight spectrum of Mu exiting a thin Ar layer. The peak at approximately $0 \mu \mathrm{s}$ arises from scattered beam positrons. (a) Without cuts, the solid line is a fitted background according to Eq. (B14) (see Sec. III D and Appendix B). The dashed and dotted curves are the two contributions to the total background. The dashed line is due to uncorrelated events in the TOF spectrum, whereas the dotted line is caused by correlated decay $e^{+}$. (b) The $\mathrm{Mu}$ TOF with the cuts described in Sec. III C. The dashed line is the fitted, uncorrelated background between 1 and $1.6 \mu \mathrm{s}$, with a fixed slope according to Eq. (B14). The total number of $\mathrm{Mu}$ atoms $N_{\mathrm{Mu}}^{\mathrm{sum}}$ is calculated by subtracting the background from the spectrum between 0.05 and $1 \mu \mathrm{s}$.

total trigger rate amounts to $0.2 / \mathrm{s}$, so that the signal to background ratio is of the order of $S / B=1 / 50$. For the $B$ field off and with cuts applied, the detected number of $\mu^{+}$is about $7.7 / \mathrm{s}$ with a total trigger rate of $15 / \mathrm{s}$.

The energy spectra $N_{\text {tot }}(E)$ and $N_{\mathrm{Mu}}(E)$ for Ar are shown in Fig. 5. The bin width $\Delta E$ at energies above $10 \mathrm{keV}$ in $N_{\mathrm{Mu}}(E)$ is determined by the bin width of $32 \mathrm{~ns}$ in the $N_{\mathrm{Mu}}(t)$ spectrum [see Eq. (2)]. At approximately $10 \mathrm{keV}$ the bin width has decreased to $2 \mathrm{keV}$. At energies below $10 \mathrm{keV}$ a fixed $\Delta E=2 \mathrm{keV}$ is chosen by integrating over several bins in the TOF spectrum. Figure 5 shows that considerable $\mathrm{Mu}$ formation sets in at energies below $15 \mathrm{keV}$, whereas, above $40 \mathrm{keV}$, practically no $\mathrm{Mu}$ is observed. A similar behavior is obtained with the other targets investigated.

\section{Cuts for background reduction}

The cuts applied to the TOF spectra are defined using the lifetime and the decay $e^{+}$energy spectra. They are shown in Figs. 6 and 7. The lifetime spectrum with the $B$ field off, in Fig. 6(a), shows a prompt peak at $0.9 \mu$ s arising from scattered beam $e^{+}$or from $\mu^{+}$, which are not detected in MCP1. 


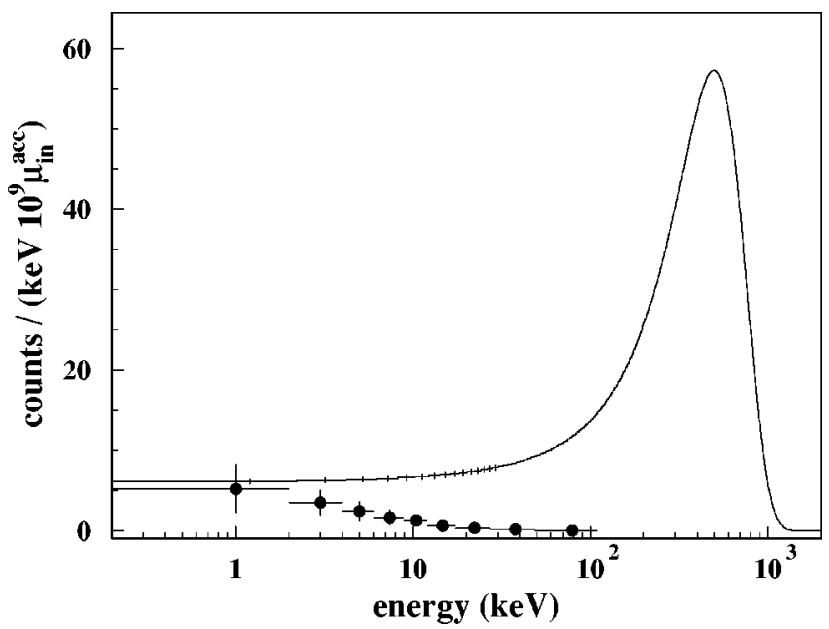

FIG. 5. Energy spectra of the muon charge states exiting a frozen Ar layer. Closed circles denote the normalized energy spectrum $N_{\mathrm{Mu}}(E)$ of $\mathrm{Mu}$, and the solid line shows the normalized energy spectrum $N_{\text {tot }}(E)$ of all muon charge states. In $N_{\text {tot }}(E)$, error bars are drawn up to $E=30 \mathrm{keV}$. The horizontal error bars in $N_{\mathrm{Mu}}(E)$ show the variable bin size in the energy spectrum.
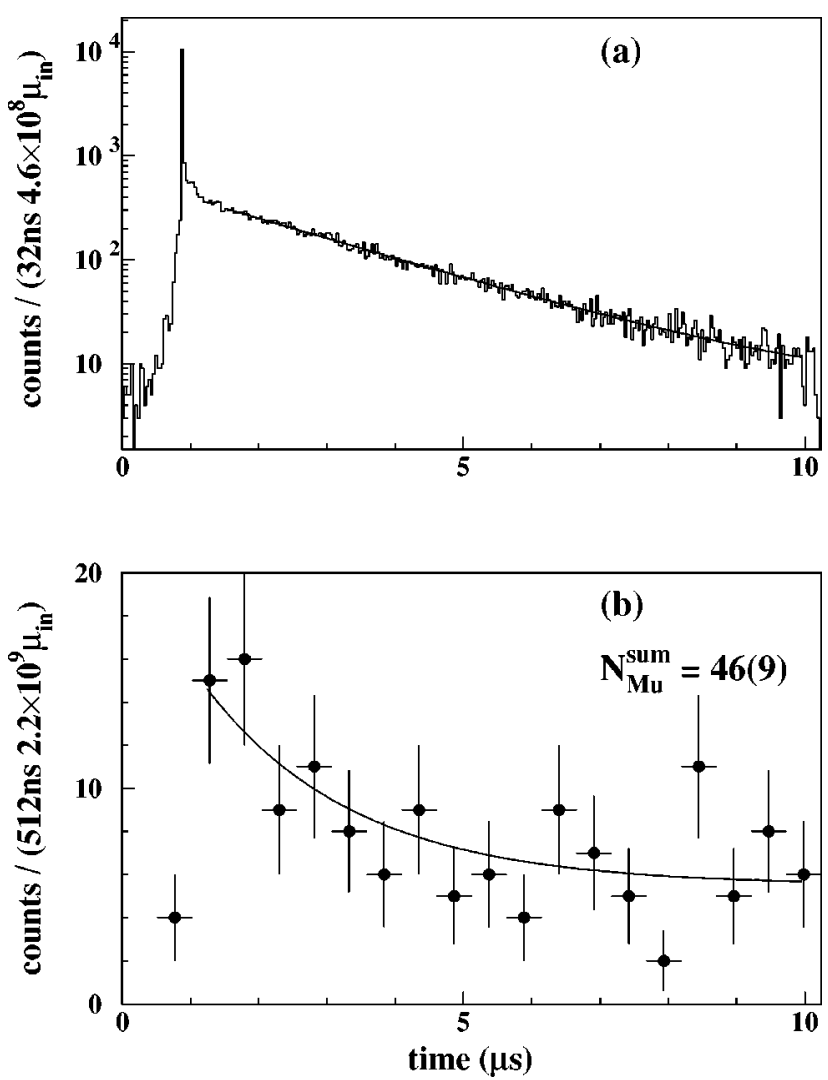

FIG. 6. (a) Lifetime spectrum without cuts ( $B$ field off). The prompt peak at $0.9 \mu \mathrm{s}$ is discussed in the text. The function of Eq. (6) is fitted using a maximum likelihood fit with Poisson statistics. The time zero $t_{0}^{d}$ is at $0.9 \mu \mathrm{s}$. The fitted lifetime $\tau=2.201(35) \mu \mathrm{s}$ is consistent with the muon lifetime $\tau_{\mu}=2.197 \mu$ s [9]. (b) Lifetime spectrum with cuts for Mu ( $B$ field on), exiting a thin Ar layer. The function of Eq. (6) is fitted to the spectrum with $\tau_{\mu}$ fixed. The total number of Mu atoms $N_{\mathrm{Mu}}^{\mathrm{sum}}$ agrees with that found in the TOF spectrum [see Fig. 4(b)].
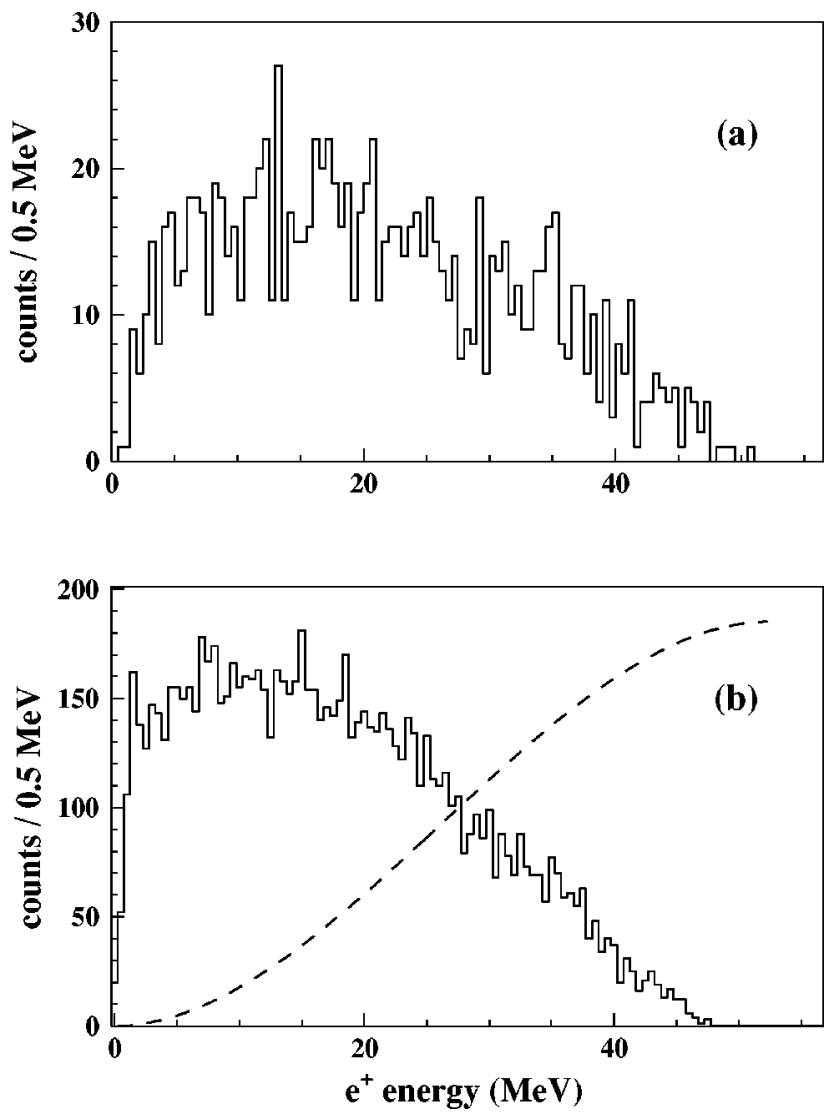

FIG. 7. (a) Measured energy spectrum in the $\mathrm{NaI}(\mathrm{Tl})$ detectors for decay positrons originating from $\mu^{+}$decay in MCP1, with the $B$ field off. (b) Energy spectrum simulated with the program GEANT [32]. The simulation allows for energy loss, straggling, and multiple scattering of the positrons in the MCP1, the vacuum tube, and the $S_{e i}$ and $\mathrm{NaI}(\mathrm{Tl})$ detectors . No detector resolution of the $\mathrm{NaI}(\mathrm{Tl})$ is included, but the simulation agrees well qualitatively with the measurement. The dashed line is the theoretical energy spectrum of the decay positrons [30].

For the latter, the subsequently emitted decay $e^{+}$gives a signal in both the MCP1 and one of the scintillator pairs at essentially the same time. In the $\mathrm{Mu}$ lifetime spectrum the origin of the prompt peak is different, and is discussed below together with the background in the Mu TOF spectra. Since these prompt events have the wrong MCP1 signal for the TOF measurement they are cut off.

The lifetime spectrum $D_{\text {tot,Mu }}\left(t^{i}\right)$ at bin $i$ is given by integrating the decay probability density with the $\mu^{+}$lifetime $\tau_{\mu}$ over each bin,

$$
\begin{aligned}
D_{\text {tot }, \mathrm{Mu}}\left(t^{i}\right) & =\int_{t_{l}^{i}} t_{h}^{i}\left[\frac{N_{\mathrm{tot}, \mathrm{Mu}}^{\mathrm{sum}}}{\tau_{\mu}} e^{-\left(t^{\prime}-t_{0}^{d}\right) / \tau_{\mu}}\right] d t^{\prime}+b \\
& =N_{\text {tot, } \mathrm{Mu}}^{\mathrm{sum}}\left[e^{\left.-\left(t_{l}^{i}-t_{0}^{d}\right) / \tau_{\mu}-e^{-\left(t_{h}^{i}-t_{0}^{d}\right) / \tau_{\mu}}\right]+b,}\right.
\end{aligned}
$$

where $t_{0}^{d}$ denotes the time zero of the decay spectrum, which is determined by the position of the prompt peak, and $b$ is a flat background per time bin assumed to be constant over the total lifetime spectrum due to the low $e^{+}$stop rate. The total number of detected muons or $\mathrm{Mu}$ atoms can be determined in an alternative way by fitting this function to the corresponding spectra. This provides a check on the correctness of 
the background subtraction in the TOF spectra, where the background is not flat over a TOF interval of a few hundred $\mathrm{ns}$. This is demonstrated by comparing the results for $\mathrm{Mu}$ of Fig. 6(b) with Fig. 4(b). Furthermore, in the case of $\mathrm{Mu}$, the lifetime spectrum convincingly demonstrates the detection of $\mathrm{Mu}$ atoms since the slope of the spectrum is consistent with the $\mu^{+}$lifetime.

Figure 7(a) shows the energy distribution of the decay $e^{+}$ originating from $\mu^{+}$decay in MCP1 detected in one of the $\mathrm{NaI}(\mathrm{Tl})$ crystals. The pulse height spectra of the crystals are calibrated by tuning the separator in the beamline to $e^{+}$, and measuring the energy deposited in the $\mathrm{NaI}(\mathrm{Tl})$ crystals by beam $e^{+}$scattered in the MCP1 detector. The energy of the beam $e^{+}$with a momentum of $27.5 \mathrm{MeV} / c$ is about 27 $\mathrm{MeV}$, which corresponds to the maximum energy deposited in the crystals. A simulated energy spectrum of decay $e^{+}$ using the program GEANT [32] is shown in Fig. 7(b), together with the theoretical spectrum (Michel spectrum) plotted as a dashed curve. The distinct deviation of the Michel spectrum from the experimental one is very well reproduced, and is a consequence of energy loss and scattering of the $e^{+}$traversing the MCP1 detector and the material surrounding MCP1. We require a minimum energy of $1 \mathrm{MeV}$ deposited in the $\mathrm{NaI}(\mathrm{Tl})$ crystals.

For the analysis of the TOF data only those events are accepted where (1) only one pair of scintillators has a hit, (2) the corresponding $\mathrm{NaI}(\mathrm{Tl})$ detector has a hit correlated in time with the scintillator pair within a time window of $40 \mathrm{~ns}$, (3) the hit is detected after the prompt peak in the lifetime spectrum, and (4) the energy deposited in the $\mathrm{NaI}(\mathrm{Tl})$ detectors is larger than $1 \mathrm{MeV}$. The effect of the applied cuts on the signal can be estimated by comparing the total number $N_{\text {tot }}^{\text {sum }}$ of detected $\mu^{+}$with and without cuts. With cuts there is a loss of signal of about $20 \%$, whereas the background is nearly completely removed.

\section{Sources of background}

We will now discuss the background in the Mu TOF spectra. Details are given in Appendix B. Without cuts, the background consists mainly of three components. The first one is due to accidental, uncorrelated coincidences of $\mu_{\text {in }}, \mathrm{MCP} 1$, and one of the scintillator pairs $S_{e i}$ within the time windows defined by the trigger electronics, depending on the single detector rates and the gate lengths. This background rate is estimated to $\sim 0.05 / \mathrm{s}$. In principle, this background is flat. However, due to the $S_{\mu}$ pile-up coincidence, which gives rise to a reset of the data acquisition, the background is modified to become exponential with a slope determined by the $S_{\mu}$ rate (see Appendix $\mathrm{B}$ ).

The other two components are caused by the prompt peak in the lifetime spectrum and are therefore correlated in MCP1 and $S_{e i}$. They consist of $e^{+}$originating from $\mu^{+}$ decays in the target region, where about $90 \%$ of the beam stops (see the simulation below), and from $e^{+}$originating from the $\mu^{+}$stopped in the side branch. The decay $e^{+}$from the target are only slightly deflected by the bending magnet (set to deflect particles of a momentum of $13 \mathrm{MeV} / c$ by $90^{\circ}$ ), because these $e^{+}$have a momentum normally higher than $13 \mathrm{MeV} / c$ [dashed curve in Fig. 7(b)]. With some probability they are scattered in MCP1 toward the direction of the $S_{e i}$ detectors (see Appendix B).

The two sources of $e^{+}$give rise to additional background in the TOF spectra. A $\mu^{+}$is detected as $\mu_{\text {in }}$, and stops in the target region or in the side branch. The corresponding decay $e^{+}$is detected in MCP1 and in one of the $S_{e i}$ detectors. The $e^{+}$signal in MCP1 $\left(e_{\mathrm{MCP} 1}^{+}\right)$starts the TOF gate in the trigger electronics (reverse timing for the TOF measurement, see Sec. II F and Fig. 2). If the delayed $\mu_{\text {in }}$ signal coincides with the TOF gate, an exponential background is observed in the TOF spectrum. The slope is given by the $\mu^{+}$decay rate $1 / \tau_{\mu}$ (again, the slope is corrected because of the $S_{\mu}$ pile-up). The $\mu_{\text {in }}$ and $e_{\text {MCP1 }}^{+}$signals are correlated. In the forward-timing TOF spectrum [see Fig. 4(a)] this background appears between time zero and $1.6 \mu \mathrm{s}$. Therefore, the only $\mu^{+}$which contribute to this background are those which decay within $1.6 \mu \mathrm{s}$ after they stopped in the apparatus. A $\mu^{+}$decaying later has a delayed $\mu_{\text {in }}$ signal outside the TOF gate. In this case a delayed $\mu_{\text {in }}$ pulse, generated by a second $\mu^{+}$, can coincide with the TOF gate. Now the $\mu_{\text {in }}$ and $e_{\text {MCP1 }}^{+}$signals are uncorrelated. The background shape is the same as in the case of accidental coincidences. Thus the background in the TOF spectra due to $e_{\mathrm{MCP} 1}^{+}$signals is a sum of two exponentials with different slopes. Since the prompt peak in the lifetime spectrum is cut off, this component of the background is removed in the analysis. The remaining background in the $\mathrm{Mu}$ TOF spectra is caused by the first component (accidental coincidences of $\mu_{\text {in }}, \mathrm{MCP} 1$, and $S_{e i}$ ) with a slope determined by the $S_{\mu}$ rate. This slope is fixed when fitting an exponential background to the Mu TOF spectra [dashed curve in Fig. 4(b)]. In Fig. 4(a) the background is fitted according to Eq. (B14). The slope is larger than for the uncorrelated case due to the additional exponential contribution from the decay $e^{+}$. The fitted, uncorrelated background is drawn as a dashed line, and the correlated one as a dotted line.

By integrating the prompt peak entries a background rate of $0.15 / \mathrm{s}$ is obtained, so that the sum with the uncorrelated background rate yields the measured trigger rate (approximately equal to the background rate) of $0.2 / \mathrm{s}$ for $\mathrm{Mu}$ data taking. Other background sources such as scattered-beam $e^{+}$ or $\mathrm{Mu}$ atoms not detected in MCP1, but their decays $e^{+}$are less intense and are also cut off in the analysis, since they contribute to the prompt peak.

\section{E. Energy-dependent neutral fraction}

The same procedure as described above for the Ar data is applied to analyze the data for $\mathrm{Al}$, solid $\mathrm{Xe}$, and $\mathrm{N}_{2}$. The background correction gives consistent results within $10 \%$. This is inferred from the comparison between the numbers $N_{\mathrm{Mu}}^{\mathrm{sum}}$ determined from the TOF and lifetime spectra. The experimental neutral fractions $\Phi_{0}(E)$ are determined using Eq. (1). They are shown in Fig. 8, and compared with the calculated fractions (solid lines). For the solid gas layers $\Phi_{0}(E)$ is calculated using Eq. (4) with the velocity scaled cross sections for protons compiled in Ref. [27]. For the Al target the solid line represents a sum of three exponentials fitted empirically by Ahn [12] to measured muon data and velocity scaled neutral fraction data of protons and deuterons $[1,33]$. The muon, proton and deuteron data used in Ref. [12] are for "dirty foils" with a thin layer of aluminum oxide at 

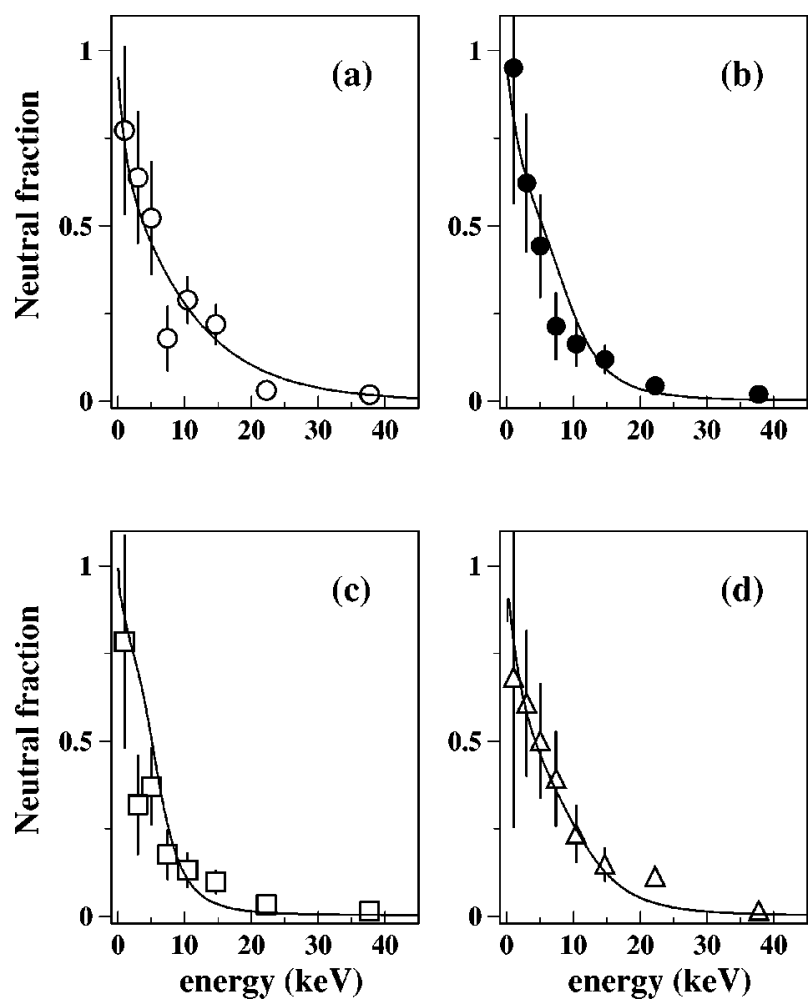

FIG. 8. Measured energy dependence of neutral fractions for $\mu^{+}$ exiting from (a) the aluminum foil ( $\mathrm{Al}$, probably covered by a few atomic layers of oxide on the surface), (b) an Ar layer on $\mathrm{Al}$, (c) a Xe layer on Al, and (d) a $\mathrm{N}_{2}$ layer on Al. The solid line in (a) is obtained from a fit by Ahn [12] to muon data and velocity scaled proton and deuteron data $[1,33]$, where the $\mathrm{Al}$ is covered by a thin layer of oxide. In (b)-(d) the solid line is obtained by a velocity scaling of cross sections for protons, and calculating the neutral fraction according to Eq. (4).

the surface. In this case the neutral fraction is different compared with a freshly evaporated Al layer [1]. Since we cannot exclude a thin film of aluminum oxide at the surface of our Al target, we use Ahn's fit to compare our data with his muon data and the scaled proton and deuteron data.

Common to all investigated targets is the decrease of $\mathrm{Mu}$ production with increasing energy. At lower energies the statistical errors increase due to the low statistics in the TOF spectra at increasing times. Within the statistical errors the data are well described by solid lines representing the scaled proton data. The results are summarized in Fig. 9, where differences between the targets become more recognizable. Figure 9(a) shows our data for the gas layers. The Al data are not drawn here to obtain a better overview. They are similar to the $\mathrm{N}_{2}$ data except the points at 8 and $22 \mathrm{keV}$, which are located below the calculated curve [see Fig. 8(a)]. There are slight differences in the shapes of the neutral fractions. The Xe data fall systematically below the Ar and $\mathrm{N}_{2}$ data. This behavior is represented in the calculated neutral fractions, shown in Fig. 9(b). For Xe the decrease of $\Phi_{0}(E)$ is steeper at lower energies. For $\mathrm{Ar}$ and $\mathrm{N}_{2}$ the calculated shape of $\Phi_{0}(E)$ is about the same above $\sim 10 \mathrm{keV}$, whereas our data for Ar are placed a little below the $\mathrm{N}_{2}$ data. In Fig. 9(b) the calculated neutral fractions yield $92 \%$ for Ar, $100 \%$ for Xe, $80 \%$ for $\mathrm{N}_{2}$, and $95 \%$ for $\mathrm{Al}$ when extrapolating to thermal energy.
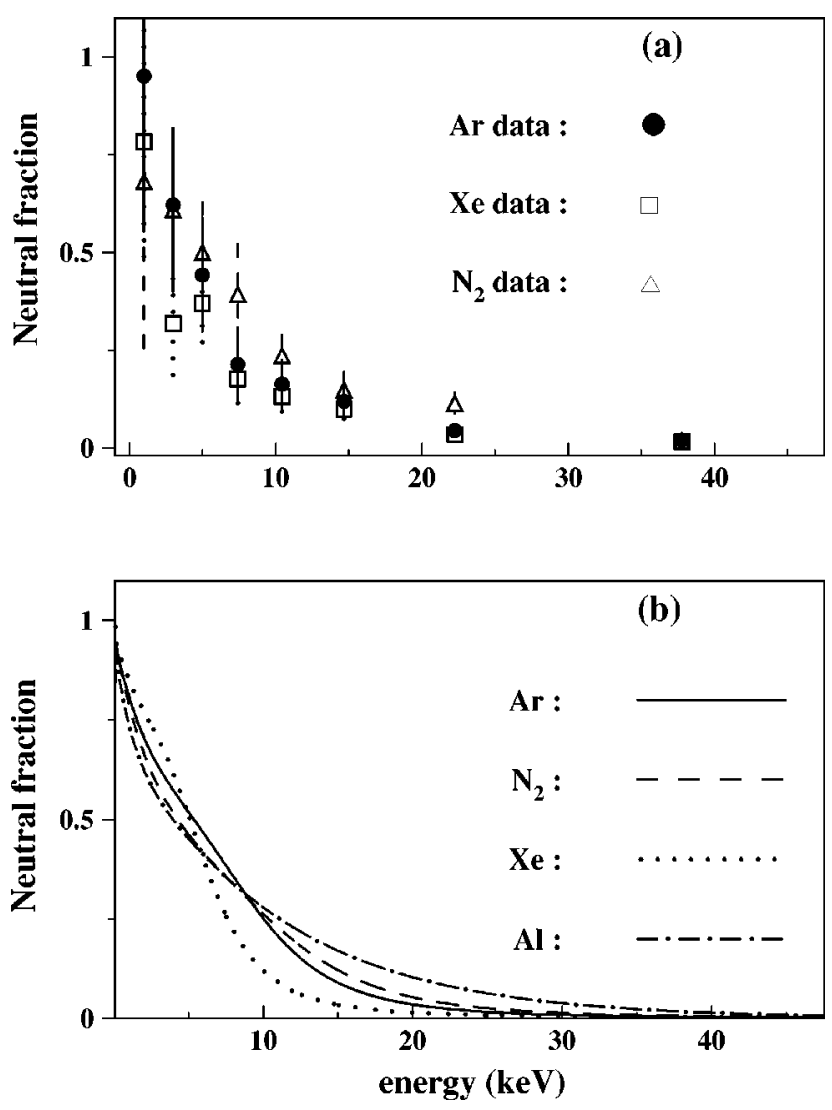

FIG. 9. (a) Measured energy-dependent neutral fraction data for $\mathrm{Ar}, \mathrm{Xe}$, and $\mathrm{N}_{2}$ targets. (b) Calculated energy dependence of the neutral fraction for $\mathrm{Al}, \mathrm{Ar}, \mathrm{Xe}$, and $\mathrm{N}_{2}$.

The different energy dependence of $\Phi_{0}(E)$ for Xe, compared with Ar and $\mathrm{N}_{2}$, can be attributed to the shift of the maximum of the electron loss cross section from $\sim 10 \mathrm{keV}$ for $\mathrm{Ar}$ and $\mathrm{N}_{2}$ to $6 \mathrm{keV}$ for Xe; see Fig. 10(b). Also, the capture cross section for $\mathrm{Xe}$ is smaller than $\sigma_{c}(E)$ for $\mathrm{Ar}$ and $\mathrm{N}_{2}$ at energies above $6 \mathrm{keV}$, see Fig. 10(a).

There is a large difference in the shape of $\sigma_{c}(E)$ below 1 $\mathrm{keV}$, which is the energy region not accessible in this experiment. The maximum of $\sigma_{c}(E)$ for $\mathrm{Xe}$ is located at $E_{\max }$ $\approx 70 \mathrm{eV}$ and for $\mathrm{Ar}$ and $\mathrm{N}_{2}$ at $E_{\max } \approx 250 \mathrm{eV}$. At an energy of $15 \mathrm{eV} \sigma_{c}(E)$ is about $1.0 \times 10^{-15} \mathrm{~cm}^{2}$ for $\mathrm{Xe}$ and about $3.0 \times 10^{-17} \mathrm{~cm}^{2}$ for $\mathrm{Ar}$ and $\mathrm{N}_{2}$. The difference in the position of the maximum can be attributed to the matching of the velocities of the $\mu^{+}$and the valence electrons in the target. For Xe, the valence electrons in the $n=5$ shell move slower than in $\operatorname{Ar}(n=3)$. This shifts the maximum of $\sigma_{c}(E)$ for Xe to lower energies. In a very simple picture the smaller value of $\sigma_{c}\left(E_{\max }\right)$ for $\mathrm{Ar}$ and $\mathrm{N}_{2}$ compared with Xe can be explained by the larger atomic cross section and the larger interaction time (due to the lower $\mu^{+}$velocity) in Xe. The smallness of $\sigma_{c}(E)$ of $\mathrm{Ar}$ and $\mathrm{N}_{2}$ compared with Xe at energies far below $E_{\max }$ can be attributed to the different ionization energies, which are $15.8 \mathrm{eV}$ for $\mathrm{Ar}, 15.6 \mathrm{eV}$ for $\mathrm{N}_{2}$, $12.1 \mathrm{eV}$ for $\mathrm{Xe}$, and $13.6 \mathrm{eV}$ for $\mathrm{Mu}$. Therefore, the capture process is energetically possible for Xe even at zero energy, whereas it is forbidden for $\mathrm{Ar}$ and $\mathrm{N}_{2}$. As well, this gives rise to the extrapolated neutral fractions of $100 \%$ for Xe and less than this for $\mathrm{Ar}$ and $\mathrm{N}_{2}$. In the solid the ionization 

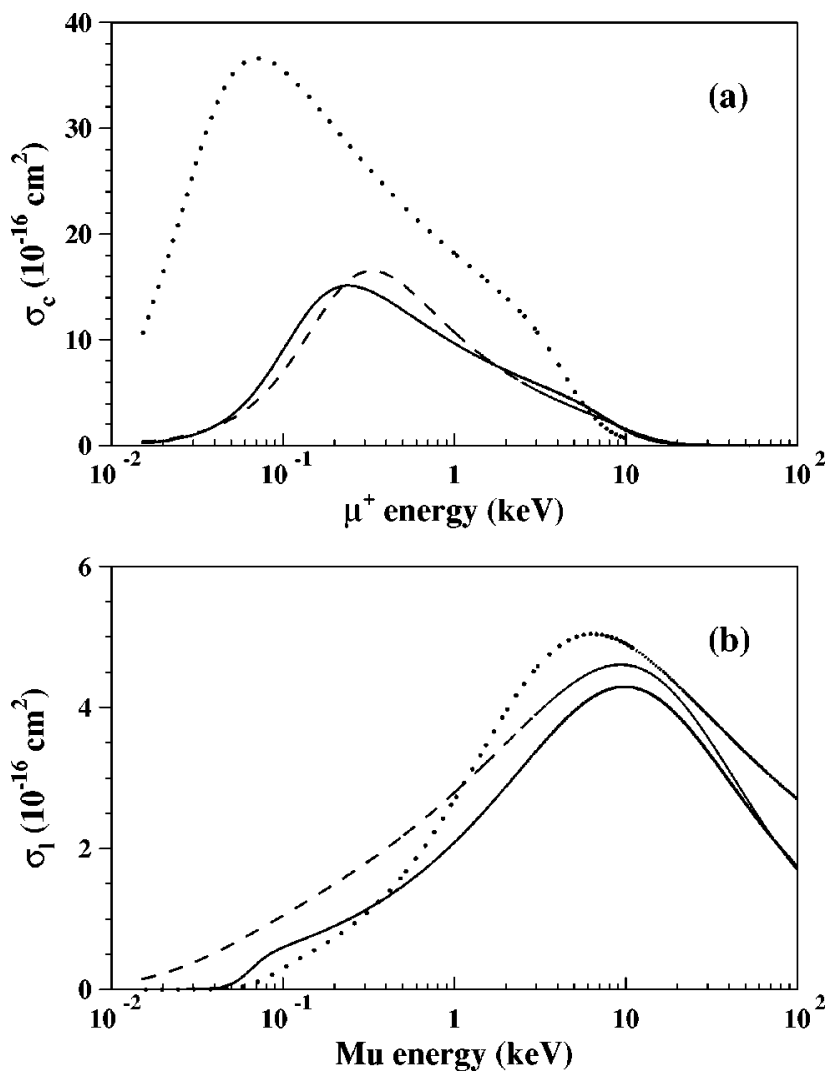

FIG. 10. Velocity scaled cross sections of protons [27] for (a) electron capture $\sigma_{c}(E)$ of $\mu^{+}$, and (b) for electron loss $\sigma_{l}(E)$ of the $\mathrm{Mu}$ atom. Solid line, Ar; dotted line, Xe; dashed line, $\mathrm{N}_{2}$.

energy has to be substituted by the band gap energy $E_{g}$ $=14.1,14$, and $9.3 \mathrm{eV}$ for $\mathrm{Ar}, \mathrm{N}_{2}$, and Xe, respectively.

\section{F. Monte Carlo simulation and integral neutral fraction}

In order to probe velocity scaling, and to estimate the integral neutral fraction $\Phi_{0}^{\text {sum }}$ in the emitted beam, we performed a Monte Carlo simulation to calculate the energy spectrum and the angular distribution of the muon beam downstream of the target. For that fraction of the beam hitting the MCP1 detector we can determine $\Phi_{0, \mathrm{MCP} 1}^{\text {sum }}$ by forming the ratio $N_{\mathrm{Mu}, \mathrm{MCP} 1}^{\mathrm{sum}} / N_{\text {tot,MCP1 }}^{\text {sum }}[$ see Eq. (5)] where we now added to the subscripts MCP1 to emphasize that this integral fraction is measured on MCP1. However, since the solid angle of MCP1 is small, the measured energy distribution of $\mu^{+}$will contain higher energies of the muon beam, because the higher the energy of a particle the lower its mean scattering angle. This means that the detected $\mu^{+}$energy distribution is shifted to higher energies compared with the energy distribution of the total exiting beam. This reduces the detected fraction of $\mu^{+}$with $E<40 \mathrm{keV}$, where $\mathrm{Mu}$ formation takes place, due to the larger mean scattering angle at lower energies. Therefore, $\Phi_{0, \mathrm{MCP} 1}^{\text {sum }}$ underestimates the integral $\mathrm{Mu}$ fraction, and the simulation is needed to calculate the energy spectrum of the total exiting beam to obtain an estimate of $\Phi_{0}^{\text {sum }}$.

The simulation takes into account the energy loss, range straggling (from which the straggling in energy loss is derived), and multiple scattering according to Molière's theory [34] in the materials the $\mu^{+}$are traversing. The energy loss
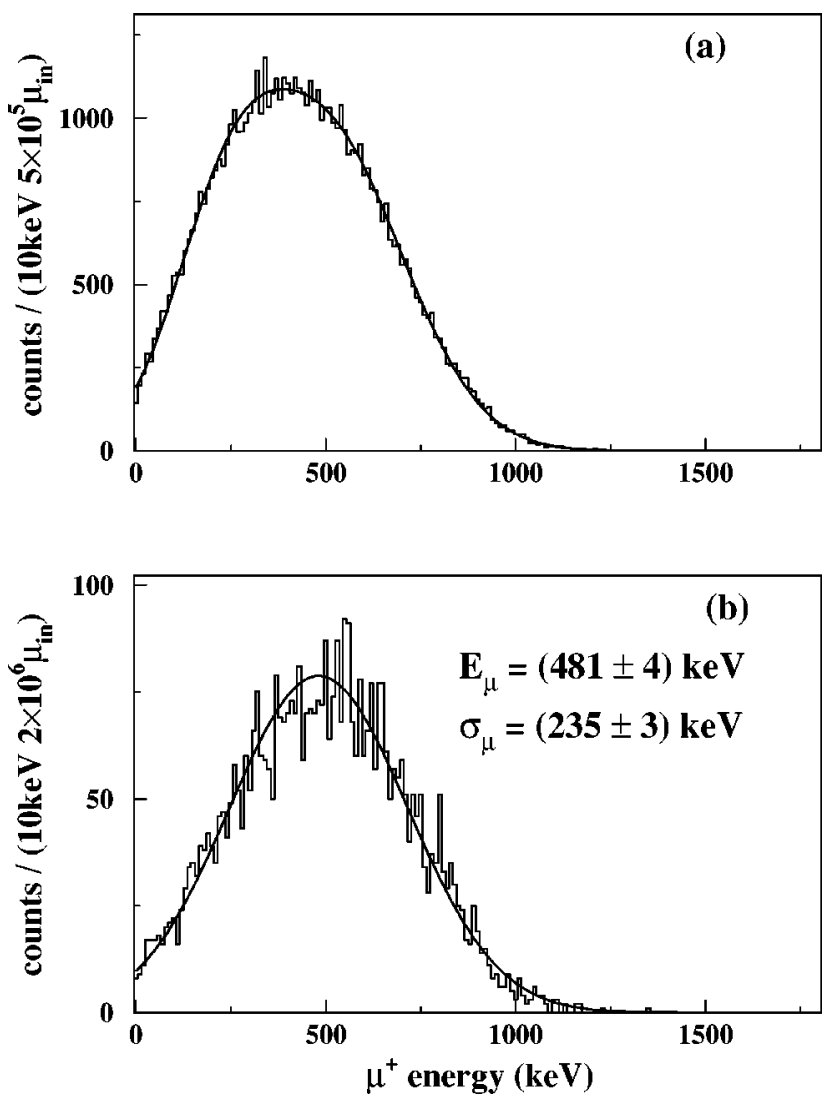

FIG. 11. Simulated energy spectra of the muon beam downstream of the target for an initial beam momentum of $p$ $=27.5 \mathrm{MeV} / c$ and $\Delta p / p=2 \%$. (a) All $\mu^{+}$exiting the target. The solid line represents a sum of two Gaussians which empirically fits well to the data. (b) Muons hitting the MCP1 detector. A Gaussian is fitted, yielding the mean energy $E_{\mu}$ and the width $\sigma_{\mu}$ of the distribution. The resulting values agree with those obtained from fitting to the experimental data (see Fig. 3) within two standard deviations.

is obtained by velocity scaling of stopping power data for protons [35], whereas the range straggling is calculated by multiplying velocity scaled proton data by $\sqrt{m_{p} / m_{\mu}}$ [36]. The velocity scaling is applied down to a $\mu^{+}$energy of 1 $\mathrm{keV}$. The $\mu^{+}$stops in the simulation if the energy becomes less than $1 \mathrm{keV}$.

The simulation shows that $87 \%$ of the incoming beam stops in the Al substrate or in the copper frame surrounding the substrate. Only $13 \%$ of the beam penetrates the target. The simulated energy spectra of the $\mu^{+}$downstream of the target and of the fraction of $\mu^{+}$hitting MCP1 are shown in Figs. 11(a) and 11(b), respectively. The energy spectrum of the total beam in Fig. 11(a) is fitted empirically by a sum of two Gaussians. The spectrum of $\mu^{+}$hitting MCP1 has a Gaussian shape, and agrees with the measured energy spectrum in Fig. 3. The simulated angular distribution of the full muon beam is shown in Fig. 12(a). The angular distribution for $\mu^{+}$with energies less than $40 \mathrm{keV}$ is broader, with a larger mean scattering angle, compared with the data including all muon energies [see Fig. 12(b)]. Therefore, the acceptance $\Omega_{\mathrm{MCP} 1}^{\mathrm{Mu}}$ of MCP1 with respect to the Mu angular distribution is smaller than $\Omega_{\text {MCP1 }}^{\text {tot }}$, which denotes the acceptance for all muon energies. The simulation yields $\Omega_{\mathrm{MCP} 1}^{\mathrm{Mu}}=1.1$ 

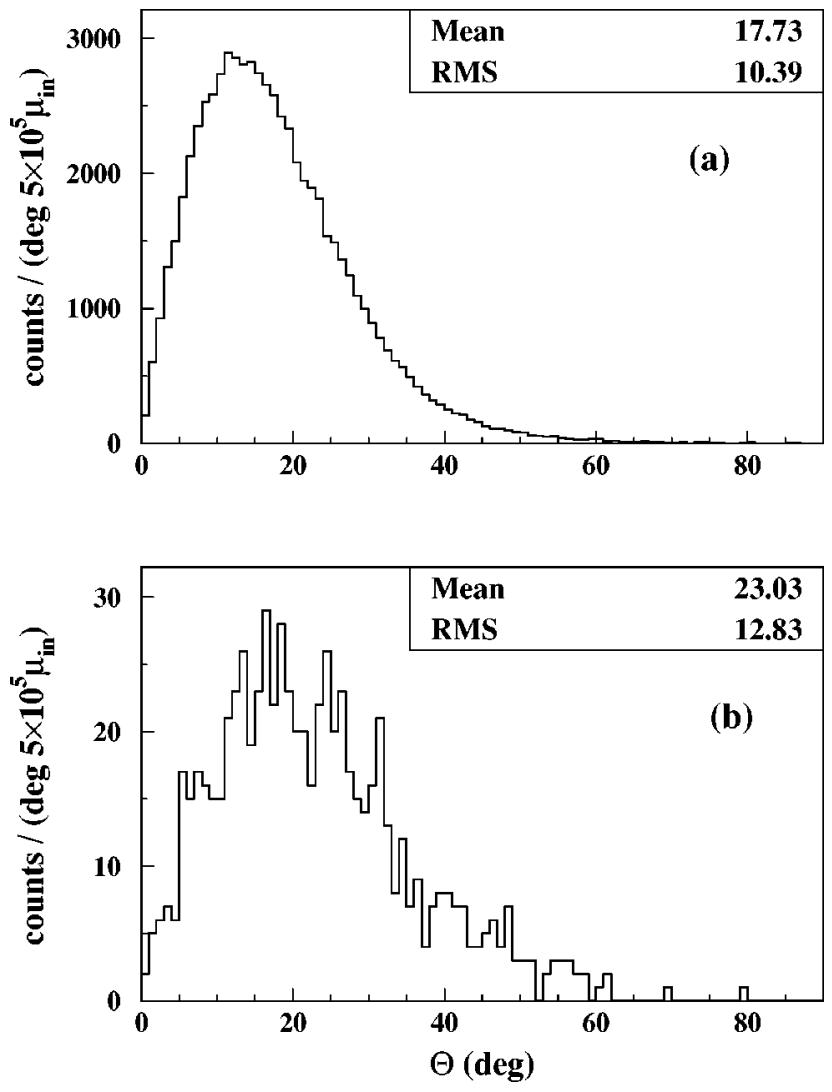

FIG. 12. Simulated angular distribution $f(\Theta) \sin (\Theta) d \Theta$ of the exiting muon beam. (a) All muons and (b) muons with energies less than $40 \mathrm{keV}$, where Mu formation sets in.

$\times 10^{-2}$ and $\Omega_{\mathrm{MCP1}}^{\mathrm{tot}}=1.7 \times 10^{-2}$. These acceptances are larger than the solid angle $\Omega_{\mathrm{MCP} 1}$ of MCP1 subtended at the target due to the forward-peaked angular distributions. The fact that $\Omega_{\mathrm{MCP} 1}^{\mathrm{Mu}}<\Omega_{\mathrm{MCP} 1}^{\mathrm{tot}}$ represents the energy dependence of the mean scattering angle and shows that $\Phi_{0, \mathrm{MCP} 1}^{\mathrm{sum}}$ will underestimate the integral fraction in the emitted beam.

The integral fractions for the different targets are summarized in Table I. The experimental fractions $\Phi_{0, \mathrm{MCP} 1}^{\mathrm{sum}}$ are well reproduced within the errors by the simulated data. The Xe data yield a smaller fraction, which is due to the reduced $\mathrm{Mu}$ formation probability at energies above $6 \mathrm{keV}$ compared with the Ar, $\mathrm{N}_{2}$, and $\mathrm{Al}$ data (see Fig. 9). The simulated integral fraction of the full beam is about $2 \times 10^{-3}$, with small deviations between the investigated targets, which again represent the slightly different $\mathrm{Mu}$ formation probabili-

TABLE I. Experimental and simulated integral neutral fractions $\Phi_{0, M C P 1}^{\text {sum }}$ of the beam hitting the MCP1 detector. Simulated data only for $\Phi_{0}^{\text {sum }}$, denoting the integral neutral fraction of the full beam downstream of the target.

\begin{tabular}{cccc}
\hline \hline Target & $\begin{array}{c}\text { Experiment } \\
\Phi_{0, \mathrm{MCP} 1}^{\text {sum }}\end{array}$ & $\begin{array}{c}\text { Simulation } \\
\Phi_{0, \mathrm{MCP} 1}^{\text {sum }}\end{array}$ & $\begin{array}{c}\text { Simulation } \\
\Phi_{0}^{\text {sum }}\end{array}$ \\
\hline $\mathrm{Ar}$ & $1.30(23) \times 10^{-3}$ & $1.36 \times 10^{-3}$ & $2.17 \times 10^{-3}$ \\
$\mathrm{Xe}$ & $1.00(20) \times 10^{-3}$ & $1.13 \times 10^{-3}$ & $1.80 \times 10^{-3}$ \\
$\mathrm{~N}_{2}$ & $1.51(30) \times 10^{-3}$ & $1.34 \times 10^{-3}$ & $2.15 \times 10^{-3}$ \\
$\mathrm{Al}$ & $1.25(15) \times 10^{-3}$ & $1.56 \times 10^{-3}$ & $2.48 \times 10^{-3}$ \\
\hline \hline
\end{tabular}

ties. The integral fractions depend on the beam momentum, the momentum bite, and the amount of material the beam has to traverse. For example, a slightly higher beam momentum shifts the mean energy of the downstream beam to higher energies. This reduces the fraction of the beam with energies below $40 \mathrm{keV}$, and therefore, $\Phi_{0}^{\text {sum }}$ is also reduced. Thus the result can be considered as a consistency check of velocity scaling with the present experimental setup. Taking into account the simulated transmission of $13 \%$ of the incoming beam the integral $\mathrm{Mu}$ formation probability per incoming $\mu^{+}$yields about $3 \times 10^{-4}$ for our setup.

\section{DISCUSSION}

In the calculation of the neutral fractions the target layers are treated as a dense atomic gas. The use of the "atomic" cross sections requires that the collision processes of the projectile with the target atoms can be considered as single collision processes. This condition is met if the time $\tau_{c}$ for a single collision process is much smaller than the average time $\tau$ between two successive impacts, $\tau_{c} / \tau \ll 1$. A crude estimate for $\tau_{c}$ is obtained by setting $\tau_{c} \approx a_{0} / v$, where $a_{0}$ is the Bohr radius, and $v$ the velocity of the projectile. The time $\tau=1 /(n v \sigma)$ is expressed by the density $n$ of atoms per $\mathrm{cm}^{3}$, the velocity $v$ of the projectile and the cross section $\sigma$. The condition for the existence of a single collision process can be written as $\tau_{c} / \tau \approx n a_{0} \sigma \ll 1$. For instance, with a density of $\rho=1.78 \mathrm{~g} / \mathrm{cm}^{3}$ for solid Ar and $\rho=3.77 \mathrm{~g} / \mathrm{cm}^{3}$ for solid Xe, the density of atoms yields $2.7 \times 10^{22}$ and $1.7 \times 10^{22} \mathrm{~cm}^{-3}$, respectively. This results in $\tau_{c} / \tau \approx \sigma \times 10^{14} \mathrm{~cm}^{-2} \ll 1$, if $\sigma$ $<10^{-15} \mathrm{~cm}^{2}$. This is fulfilled for $\mu^{+}$energies greater than 1 $\mathrm{keV}$ (see Fig. 10). Also, the de Broglie wavelength $\lambda$ of the $\mu^{+}$is required to be less than the interatomic distance in order to treat the projectile as a pointlike particle during the collision process. For $\mu^{+}$with an energy of $1 \mathrm{keV}$ the wavelength $\lambda=2.7 \times 10^{-12} \mathrm{~m}$ is about 100 times smaller than interatomic scales. The considerations above show that the simple picture of single collisions within the solid is well justified for the condensed gas targets.

At $\mu^{+}$energies below approximately $10 \mathrm{keV}$, corresponding to $\mu^{+}$velocities $v<2 v_{e}\left(v_{e}\right.$ is the velocity of the bound electrons), solid-state effects like dynamic screening, and resonant and Auger processes as well as tunneling of electrons to the projectile leaving the surface, may affect the charge-transfer reactions [37-39]. These processes are attributed to a uniform gas of free electrons in the solid. These solid-state effects have to be taken into account to yield good agreement between measured and calculated charge fractions of protons emerging from metal or carbon foils. However, the assumption of a uniform electron gas does not hold for wide-band-gap insulators like rare-gas solids or solid $\mathrm{N}_{2}$, where all electrons are tightly bound in the valence band. Nevertheless, during slowing down the $\mu^{+}$creates an ionization track of free electrons in the conduction band and, from recent measurements on thermalized $\mu^{+}$in solid $\mathrm{N}_{2}$ and solid rare gases, it is known that these electrons have a high mobility [20]. However, the track electrons cannot behave as an electron cloud moving with the projectile, because the $\mu^{+}$ moves "in front of" its own radiolysis electrons. Therefore, the solid-state effects are expected to have no significant influence on the neutral fraction at low energies in the con- 
densed van der Waals solids. This is in accordance with our experimental results where the measured neutral fractions are described satisfactorily by the calculated fractions using the scaled cross sections.

\section{A. Velocity scaling down to $1 \mathrm{keV}$, and $Z$ dependence of the neutral fraction}

From our data we can conclude that, within the statistical error, a velocity scaling of charge-exchange cross sections is applicable for $\mu^{+}$with energies down to $1 \mathrm{keV}$, corresponding to an energy per nucleon of about $9 \mathrm{keV} /$ nucleon. This extends the available data of charge-exchange processes for "hydrogen isotopes" from the energy interval above $8 \mathrm{keV}$ for deuterons $[5,33]$ to lower energies. Also, the underlying assumption of velocity scaling of the stopping power and the range straggling of energetic $\mu^{+}$traversing the target seems well confirmed by the good agreement between the Monte Carlo results and the measured energy distribution and integral neutral fractions. This implicitly demonstrates that not only charge-exchange processes, but also ionization, which is the dominant process in energy loss at $\mu^{+}$energies above $\sim 5 \mathrm{keV}$, scales with velocity. Additionally, the good agreement between measured and simulated energy spectra implies the proper handling of multiple scattering for the $\mu^{+}$in the traversed material. According to Molière's theory the scattering angle is proportional to $1 /(p v)$, with $p$ the momentum and $v$ the velocity of the projectile.

The small deviations in the shapes of $\Phi_{0}(E)$ for the different solid gas layers are attributed to the $Z$ (atomic number) dependence of the capture and loss cross sections. For the rare-gas data compiled in Ref. [27], with increasing $Z$, both the capture and loss cross sections increase at low energies with a shift of the maximum $E_{\max }$ to lower energies. At energies larger than $E_{\max }$ only the loss cross section increases significantly with $Z$.

The dependence on $Z$ of the energy-dependent as well as integral neutral fraction is not expected to be monotonic. Recent measurements with protons and boron as projectiles on several metallic elements ranging from $\mathrm{Be}$ to $\mathrm{Au}$ show $Z$-dependent oscillations at energies of $50-500 \mathrm{keV} /$ nucleon, reflecting the periodic shell structure of the target atoms [40]. In Ref. [40] the cross sections $\sigma_{c}(E)$ and $\sigma_{l}(E)$ are calculated to describe the data qualitatively, using the Oppenheimer-Brinkmann-Kramers formula for $\sigma_{c}(E)$ and the classical-trajectory Monte Carlo method for $\sigma_{l}(E)$. The calculation was done for proton energies of $200 \mathrm{keV}$, where the simple picture of single collisions of protons in metals without solid-state effects is applicable [28]. The calculation shows that the $Z$ oscillations originate from the capture cross sections, whereas the loss cross sections depend monotonically on $Z$. From the data in Ref. [40] the neutral fraction of protons for solid Ar and Xe can be interpolated linearly between adjacent data points of metallic elements. For each of their three plotted energies of 50, 100, and $200 \mathrm{keV} /$ nucleon the interpolation of $\Phi_{0}(E)$ yields a higher neutral fraction for Xe than for Ar. This is in contradiction to the result obtained using the compiled cross sections for protons, as well as to our measurements for $\mu^{+}$, which are well described by the scaled cross sections. The discrepancy is probably due to an additional structure in the $Z$ dependence of
$\Phi_{0}(E)$, which was not resolved in the experiment of Ref. [40], where condensed gases could not be studied. According to Ref. [40] the largest $\mathrm{Mu}$ yield can be expected for medium- $Z$ elements like copper or zinc. For instance, at 50 $\mathrm{keV} /$ nucleon, corresponding to a $\mu^{+}$energy of $5.6 \mathrm{keV}$, a neutral fraction of about $50 \%$ is found for $\mathrm{Cu}$ or $\mathrm{Zn}$, and $35 \%$ for Al. These results were obtained with clean surfaces. The solid curve in Fig. 8(a), which fits well to our Al data, yields a larger neutral fraction of $42 \%$ at a $\mu^{+}$energy of $5.6 \mathrm{keV}$. The curve represents a fit to the data of oxidized Al surfaces. Compared with a clean Al surface the oxide layer causes an increase of the neutral fraction above $40 \mathrm{keV} /$ nucleon. Below $40 \mathrm{keV} /$ nucleon the neutral fraction is larger for the clean surface [1].

\section{B. Velocity scaling and moderation of $\mu^{+}$below $1 \mathrm{keV}$}

As mentioned in Sec. I, our work was also motivated by the desire to obtain a better insight into the details of the mechanisms leading to the emission of epithermal $\mu^{+}$from solid van der Waals layers. Relevant to this question is the slowing down of $\mu^{+}$and the corresponding cross sections at $\mu^{+}$energies $E<1 \mathrm{keV}$. At these energies our experiment is not sensitive to the detection of $\mu^{+}$or $\mathrm{Mu}$, since the detection efficiency of the MCP1 detector decreases rapidly below $1 \mathrm{keV}$. No direct measurements are available in this case. However, some results have been obtained by considering the thermalization time of $\mu^{+}$and its influence on the muon spin rotation $(\mu \mathrm{SR})$ signal of stopped $\mu^{+}$in gases at various pressures $[18,19]$. In a weak transverse magnetic field the precession of the $\mu^{+}$can easily be distinguished from the precession of the $\mathrm{Mu}$ atom, which is approximately 100 times faster. The $\mu \mathrm{SR}$ method can therefore measure the fraction of $\mu^{+}$thermalizing as Mu. Also, the total slowing down time as well as the time spent in the ionization, the charge-cycling and the elastic collision regime can be estimated using scaled cross sections for protons and compared with the experimental finding. It is the time spent in the charge-cycling regime which influences the observable muon polarization after thermalization. The $\mathrm{Mu}$ states formed initially from $100 \%$ polarized $\mu^{+}$by capture of unpolarized electrons are, with equal probability, the parallel $\left|\alpha_{\mu} \alpha_{e}\right\rangle$ and antiparallel $\left|\alpha_{\mu} \beta_{e}\right\rangle$ states. Since the $\left|\alpha_{\mu} \beta_{e}\right\rangle$ state is not an eigenstate of the hyperfine interaction Hamiltonian in the $\mathrm{Mu}$ atom, but a mixture of the singlet and triplet states, it oscillates with the hyperfine frequency of $4.463 \mathrm{GHz}$ between the $\left|\alpha_{\mu} \beta_{e}\right\rangle$ and $\left|\beta_{\mu} \alpha_{e}\right\rangle$ states, which gives rise to a depolarization of the $\mu^{+}$. If the total $\mathrm{Mu}$ residence time during moderation is of the order of the hyperfine period, a large amount of polarization is lost. The lower the pressure the larger the residence time in the $\mathrm{Mu}$ state and, therefore, the observed polarization decreases with decreasing pressure. The observed, pressure-dependent polarization in Ref. [18] is in good agreement with a calculation using scaled cross sections for charge exchange down to $E \sim 50 \mathrm{eV}$.

The measured neutral fractions in Ref. [18] at thermal energy in gases are 74(4)\%, 100(4)\%, and 84(4)\% for Ar, $\mathrm{Xe}$, and $\mathrm{N}_{2}$, respectively. This has to be compared with our scaled values of $92 \%, 100 \%$ and $80 \%$ (see Sec. IIIE). The agreement is good with the exception of the Ar data. A more detailed analysis by Senba [19] of the moderation process in 
Ar below $1 \mathrm{keV}$, accounting also for elastic energy loss and energy loss by ionization, estimated a Mu fraction at thermal energies of $74 \%$, again using the velocity-scaled cross sections for charge exchange and energy-scaled cross sections for the elastic process. This differs from the steady-state fraction of $92 \%$ obtained from Eq. (4) because this fraction does not account for energy-loss mechanisms. No estimations are published for Xe and $\mathrm{N}_{2}$. The steady-state fraction can be considered as the fraction of time the $\mu^{+}$spends in a charge state. It is an appropriate approximation for the measured neutral fraction after passing through a target, if the target thickness allows for a sufficient number of collisions to obtain the charge-state equilibrium and if the thickness does not effect appreciably the energy of the projectile. This last condition is always met for a transmission experiment since, for instance, at an energy of $10 \mathrm{keV}$ the steady state is obtained within a few atomic layers below the surface where the energy loss is negligible. Senba's work focused on the charge states during slowing down at fixed energy, considering the probabilities for the projectile to be in the charged (neutral) state or to change the charge state from positive to neutral (neutral to positive). At some fixed energy the estimated probability of having the particle in the neutral state does not correspond to the steady-state fraction, but the estimate at thermal energy agrees well with the neutral fractions observed as well as with the pressure-dependent polarization of $\mu^{+}$stopped in gases.

The experimental $\mu$ SR data at thermal energies are well interpreted by the cross sections for charge exchange of protons scaled to $\mu^{+}$energies $E<10 \mathrm{eV}$, although there are uncertainties in the cross sections of protons for energies less than $100 \mathrm{eV} /$ nucleon due to missing experimental data in this energy regime. This is in accordance with some calculations concerning the electron capture of $\mu^{+}+\mathrm{H}$ collisions [41]. The calculations are performed using the impact parameter model, where the relative motion of the particles is treated classically, and which is known to give a reasonable description of the $p+\mathrm{H}$ system in the velocity range corresponding to energies of $100 \mathrm{eV} /$ nucleon up to $100 \mathrm{keV} /$ nucleon [42]. The work of Ref. [41] shows that the electron capture process into the ground state of $\mathrm{Mu}$ scales with velocity down to $E \sim 15 \mathrm{eV}$, whereas for the capture into excited states and also for the cross section of excitation, velocity scaling at $E<300 \mathrm{eV}$ does not hold. Since the total inelastic cross section is dominated by the capture into the ground state, velocity scaling is applicable down to $\sim 15 \mathrm{eV}$. A deviation (isotopic effect) of the velocity dependence of charge-changing cross sections for this system (where $p$ and $\mathrm{H}$ may be substituted by the corresponding deuteron isotopes $\mathrm{D}^{+}$and $\mathrm{D}^{0}$, or by $\mu^{+}$and $\mathrm{Mu}$ ) is expected only at energies below $2 \mathrm{eV} /$ nucleon due to the slight isotopic shift in the binding energies in $\mathrm{H}, \mathrm{D}^{0}$, and $\mathrm{Mu}$ [43].

The $p+\mathrm{H}$ is a special symmetric collision system for which theoretical considerations cannot be generalized. In our case of nonsymmetric systems, at least as far as charge exchange and ionization processes are concerned, deviations from velocity scaling can be expected at energies of the order of $100 \mathrm{eV} /$ nucleon, corresponding to $E \sim 10 \mathrm{eV}$ for $\mu^{+}$ (however, at an energy of $10 \mathrm{eV}$ the electron-loss cross section for $\mathrm{Mu}$ is zero due to the ionization energy of $13.6 \mathrm{eV}$ for $\mathrm{Mu}$, and therefore, velocity scaling cannot hold. For hy- drogen with the same velocity, corresponding to an energy of $\sim 100 \mathrm{eV} /$ nucleon, this process is still allowed with a nonzero cross section). This is the relevant energy range for the processes leading to epithermal $\mu^{+}$emission from solid rare gas and $\mathrm{N}_{2}$ layers. Our moderation studies show that the epithermal $\mu^{+}$are emitted from an Ar layer with a mean energy of $10 \mathrm{eV}$, with a FWHM of $20 \mathrm{eV}$. The measured fraction of slow $\mu^{+}$per incoming muon is $4.7 \times 10^{-5}$ for $\mathrm{Ar}$, $3.3 \times 10^{-5}$ for $\mathrm{N}_{2}$ and $0.02 \times 10^{-5}$ for Xe, respectively; for Ar, a large escape depth of $\sim 100 \mathrm{~nm}$ is found [26], which is a direct manifestation of the strongly reduced electronic stopping power at low energies in this system. Using the scaled cross section $\sigma_{c}(E)$ the mean free path length $\lambda$ $=1 /\left[n \sigma_{c}(E)\right]$ for a capture process in Ar is estimated to $\lambda$ $=40 \mathrm{~nm}$ at $E=10 \mathrm{eV}\left[\sigma_{c}(E)=9 \times 10^{-18} \mathrm{~cm}^{2}\right]$ and $\lambda$ $=120 \mathrm{~nm}$ at $E=6 \mathrm{eV}\left[\sigma_{c}(E)=3 \times 10^{-18} \mathrm{~cm}^{2}\right]$. This corresponds well to the observed escape depth.

In his analysis of the $\mu$ SR data of $\mu^{+}$stopped in gaseous $\mathrm{Ar}$ and $\mathrm{N}_{2}$, Senba estimated a mean energy for the last $\mathrm{Mu}$ formation of 10(5) eV which is well above the inelastic threshold of $2.2 \mathrm{eV}(0.5 \mathrm{eV})$ for the capture process in Ar gas (solid). The Mu formed at this energy thermalizes by elastic collisions and does not undergo any further charge-changing cycles $[18,19]$. Applying the result obtained for the gas target to the solid, one may conclude that the mean emission energy of the epithermal $\mu^{+}$corresponds to the mean energy of the last Mu formation. Since there is no sharp threshold near this energy, the energy distribution of the emitted $\mu^{+}$is smeared out. Practically no slow $\mu^{+}$emitted from Ar are observed with $E>50 \mathrm{eV}$, because here charge-changing processes are still present to moderate the $\mu^{+}$efficiently. The contribution of elastic processes to the stopping power $d E / d x$ is expected to be small. The elastic stopping power for isotropic scattering, $(d E / d x)_{\mathrm{el}}=-2 m_{\mu} / M E n \sigma_{\mathrm{el}}(E)$, with the $\mu^{+}$mass $m_{\mu}$, the target mass $M$, the number density of the moderator $n$, and the cross section $\sigma_{\mathrm{el}}(E)$, yields a relative energy loss of only $0.5 \%$ for each elastic process. In $100 \mathrm{~nm}$ of solid Ar this gives roughly a relative energy loss of about $10 \%$ for typical values of $\sigma_{e l}(E)$ $\sim 10^{-16} \mathrm{~cm}^{2}$ below $100 \mathrm{eV}$ [19].

The emitted fraction of $\mu^{+}$appears to be determined by the $\mathrm{Mu}$ formation probability. Using the $\mathrm{Mu}$ fractions measured in gases at thermal energy one estimates a remaining fraction of $\mu^{+}$at energies of $\sim 10 \mathrm{eV}$ of 26(4)\%, 16(4)\%, and $0(4) \%$ for $\mathrm{Ar}, \mathrm{N}_{2}$, and $\mathrm{Xe}$, respectively. The relative fractions of the slow $\mu^{+}$yields agrees within the errors with the relative fractions of remaining $\mu^{+}$:

$$
\frac{3.3 \times 10^{-5}}{4.7 \times 10^{-5}}=0.7 \approx 0.6(18)=\frac{16(4) \%}{26(4) \%} \text { for } \mathrm{N}_{2}-\mathrm{Ar},
$$

and

$$
\frac{0.02 \times 10^{-5}}{4.7 \times 10^{-5}}=0.004 \approx 0(0.15)=\frac{0(4) \%}{26(4) \%} \text { for Xe-Ar. }
$$

However, the absolute yields of slow $\mu^{+}$are not well reproduced within this simple picture. Our Monte Carlo simulation shows that a fraction of $\sim 10^{-3}$ of the incoming beam stops in a 100-nm layer of solid Ar. This fraction should be 
comparable to the fraction of $\mu^{+}$moving with epithermal energies through the solid. Accounting for the loss due to $\mathrm{Mu}$ formation and assuming an isotropic angular distribution of the slow $\mu^{+}$within the solid after passing through some tens of $\mathrm{nm}$, yielding an escape probability of $50 \%$, one estimates a slow $\mu^{+}$fraction of about $1.3 \times 10^{-4}$ for Ar which is about three times larger than the observed fraction. Other processes like hot-atom reactions of $\mathrm{Mu}$ (for example, formation of the $\mathrm{ArMu}^{+}$molecular ion) and reactions with spur electrons are not considered here. From $\mu$ SR studies it is well known that these processes have to be taken into account to interpret the observed charge fractions and polarization losses in highpressure gases as well as in the condensed phases $[44,45,20]$. For example, the Mu fraction of 91(9)\% found in solid $\mathrm{Ar}$ [46] is larger than the fraction in the gas. This can be explained by the convergence of a $\mu^{+}$after thermalization with a spur electron which, however, should have no influence on the epithermal $\mu^{+}$. Hot-atom reactions of $\mathrm{Mu}$ are believed to occur when the $\mathrm{Mu}$ is leaving the charge-exchange regime [18], which is below $50 \mathrm{eV}$ for the targets investigated. They should reduce the yield of epithermal $\mu^{+}$in the solid van der Waals layers.

For a more precise understanding of the behavior of $\mu^{+}$ in these solids in the "experimental energy gap" between thermal energy and $1 \mathrm{keV}$, concerning especially the scaling of the charge-exchange cross sections, future investigations are possible with the slow $\mu^{+}$beam developed at PSI, where the energy of the $\mu^{+}$can be tuned by electrostatic acceleration between approximately $10 \mathrm{eV}$ and $30 \mathrm{keV}$.

\section{CONCLUSION}

We have measured the neutral fraction of a $\mu^{+}$beam at energies between 1 and $40 \mathrm{keV}$, exiting cryogenic insulators such as $\mathrm{Ar}, \mathrm{Xe}$, and $\mathrm{N}_{2}$. The data are compared with velocity-scaled cross sections for electron capture and electron loss of protons in gaseous $\mathrm{Ar}, \mathrm{Xe}$, and $\mathrm{N}_{2}$. Within the statistical errors our data agree with the result obtained from the scaled cross sections. The integral yield of $\mathrm{Mu}$ from solid $\mathrm{Ar}$ and $\mathrm{N}_{2}$ layers is comparable to that from $\mathrm{Al}$; the yield from solid Xe is about $20 \%$ smaller. This is well reproduced by a Monte Carlo simulation. Recent measurements with protons suggest that medium- $Z$ elements like copper or zinc are the best suited to produce $\mathrm{Mu}$ with energies of a few $\mathrm{keV}$.

Due to their simplicity van der Waals solids can be treated as a dense atomic gas in order to extrapolate data from gases to the solid state. In order to close the "experimental gap" for $\mu^{+}$, concerning inelastic and elastic processes between thermal energy (gas and solid data) and energies above 1 $\mathrm{keV}$ (solid data, this work), this feature was used for the discussion of velocity scaling of charge-exchange cross sections and the moderation of $\mu^{+}$leading to epithermal $\mu^{+}$ emission from these layers. The discussion showed that velocity scaling should be applicable down to $\mu^{+}$energies of approximately $10 \mathrm{eV}$. The fraction and mean energy of epithermal $\mu^{+}$emitted from the solid layers appears to be mainly determined by epithermal $\mathrm{Mu}$ formation in the layers.

\section{ACKNOWLEDGMENTS}

One of us (T.P.) gratefully acknowledges financial support from the Land Baden-Württemberg (Germany) and by a grant from the Human Capital and Mobility Program (European Community) and the Swiss Federal Office of Education and Science. We also gratefully acknowledge the help of D. C. George of the magnet group at PSI for the construction of the bending magnet, and of D. Maden from PSI, who set up the software of the data acquisition system. Finally, we wish to thank the people involved at PSI and Heidelberg for their support of this experiment.

\section{APPENDIX A: TIME-OF-FLIGHT SPECTRUM OF A GAUSSIAN ENERGY DISTRIBUTION}

A Gaussian energy spectrum with a mean energy $E_{\mu}$ and a width $\sigma_{\mu}$ of the form

$$
N(E)=\frac{N^{\text {sum }}}{\sqrt{2 \pi} \sigma_{\text {tot }}} e^{-\left(E-E_{\mu}\right)^{2} / 2 \sigma_{\mu}^{2}}
$$

is related to its time spectrum via Eq. (2). The normalization in Eq. (A1) is valid for integration from $[-\infty, \infty]$. However, in the case of only positive energies the normalization must be done for an energy interval of $[0, \infty]$. Therefore, Eq. (A1) must be modified to

$$
N(E)=\frac{N^{\text {sum }}}{\sqrt{\frac{\pi}{2} \sigma_{\mu} \operatorname{erfc}\left(-\frac{E_{\mu}}{\sqrt{2} \sigma_{\mu}}\right)}} e^{-\left(E-E_{\mu}\right)^{2} / 2 \sigma_{\mu}^{2},}
$$

with the complementary error function erfc defined as

$$
\operatorname{erfc}(x)=\frac{2}{\sqrt{\pi}} \int_{x}^{\infty} e^{-s^{2}} d s .
$$

The time spectrum $N_{\text {tot }}\left(t^{i}\right)$ at bin $i$ is given by integration over the bin width with the lower and upper bounds $t_{l}^{i}$ and $t_{h}^{i}$, respectively:

$$
\begin{aligned}
N_{\mathrm{tot}}\left(t^{i}\right) & =\int_{t_{l}^{i}}^{t_{h}^{i}}\left[N_{\mathrm{tot}}\left(E\left(t^{\prime}\right)\right)+B\left(t^{\prime}\right)\right] d t^{\prime} \\
& =C \int_{E_{l}^{i}}^{E_{h}^{i}} e^{-\left(E-E_{\mu}\right)^{2} / 2 \sigma_{\mu}^{2}} d E+B \Delta t,
\end{aligned}
$$

where we have inserted Eq. (A2) for $N(E), C$ represents the normalization factor, $\Delta t$ denotes the bin width in the time spectrum, and $E_{h}^{i}$ and $E_{l}^{i}$ are the energies corresponding to the bin boundaries $t_{h}^{i}$ and $t_{l}^{i}$ in the time spectrum. The integral over energy can be evaluated easily by a variable substitution $s \equiv\left(E-E_{\mu}\right) / \sqrt{2} \sigma_{\mu}$ yielding

$$
\begin{aligned}
\int_{E_{l}^{i}}^{E_{h}^{i}} e^{-\left(E-E_{\mu}\right)^{2} / 2 \sigma_{\mu}^{2}} d E & =\sqrt{2} \sigma_{\mu} \int_{x_{h}^{i}}^{x_{l}^{i}} e^{-s^{2}} d s \\
& =\sqrt{2} \sigma_{\text {tot }} \frac{\sqrt{\pi}}{2}\left[\operatorname{erf}\left(x_{l}^{i}\right)-\operatorname{erf}\left(x_{h}^{i}\right)\right],
\end{aligned}
$$


with the error function

$$
\operatorname{erf}(x)=\frac{2}{\sqrt{\pi}} \int_{0}^{x} e^{-s^{2}} d s,
$$

and the integration limits from the variable substitution:

$$
\begin{gathered}
x_{l}^{i}=\frac{E_{h}^{i}-E_{\mu}}{\sqrt{2} \sigma_{\mu}}, \\
x_{h}^{i}=\frac{E_{l}^{i}-E_{\mu}}{\sqrt{2} \sigma_{\mu}} .
\end{gathered}
$$

Inserting Eq. (A5) into Eq. (A4) just gives the result of Eq. (3):

$$
N_{\text {tot }}\left(t^{i}\right)=\frac{N_{\text {tot }}^{\text {sum }}}{\operatorname{erfc}\left(-\frac{E_{\mu}}{\sqrt{2} \sigma_{\mu}}\right)}\left[\operatorname{erf}\left(x_{l}^{i}\right)-\operatorname{erf}\left(x_{h}^{i}\right)\right]+B \Delta t .
$$

Using the relativistic energy relation for the kinetic energy $E_{\text {kin }}=(\gamma-1) m c^{2}$, Eq. (A7) can be rewritten to read

$$
x_{l, h}^{i}=\frac{1}{\sqrt{2} \sigma_{\mathrm{tot}}}\left[\left(\frac{1}{\sqrt{1-\left(l / c t_{l, h}^{i}\right)^{2}}}-1\right) m c^{2}-E_{\mu}\right] .
$$

The subscripts are chosen in such a manner that $x_{l}$ corresponds to the lower time bin boundary, $t_{l}$ and $x_{h}$ to the upper one $t_{h}$.

\section{APPENDIX B: BACKGROUND SHAPE AND RATE IN THE MU TIME-OF-FLIGHT SPECTRA}

In a TOF measurement, accidental coincidences within some time window $\Delta_{\mathrm{TOF}}$ in the two independent detectors for the start and stop signal may occur. If there are no correlated processes and if the detector rates are assumed to be constant per unit time, the probability density $f(t)$ of having the first stop event at time $t$ with a stop rate $R$ is given by the exponential distribution [47]

$$
\begin{array}{rlrl}
f(t) & =R e^{-R\left(t-t_{0}\right)} \equiv f^{+}(t) & & \left(\text { for } t \in\left[t_{0},+\infty\right]\right) \\
& =R e^{+R\left(t-t_{0}\right)} \equiv f^{-}(t) \quad\left(\text { for } t \in\left[-\infty, t_{0}\right]\right),
\end{array}
$$

where the first detector defines the starting time $t=t_{0}$. Equation (B1) also holds for the probability density of having an event in a detector at time $t$ preceded (or followed) by an event in the same detector at time $t=t_{0}$. This is a feature of the exponential distribution, which has "no memory" about what happened in the past and therefore, $f(t)$ is independent on the event defining the time zero.

The probability $p(t)$ of having a stop event (or an event in the same detector given another event at $t=t_{0}$ ) in the time interval $[t,(t+d t)]$, with $d t$ infinitesimal, is given by

$$
\begin{array}{cc}
p^{+}(t)=f^{+}(t) d t=R e^{-R\left(t-t_{0}\right)} d t & \left(\text { for } t \in\left[t_{0},+\infty\right]\right), \\
p^{-}(t)=f^{-}(t) d t=R e^{+R\left(t-t_{0}\right)} d t & \left(\text { for } t \in\left[-\infty, t_{0}\right]\right) .
\end{array}
$$

Using Eq. (B2) we can calculate the probability $P^{+}\left(t_{1}\right.$ $+\Delta t$ ) of obtaining the first uncorrelated event at time $t_{1}$ $\in\left[t_{0},+\infty\right]$ in a time bin with width $\Delta t$

$$
\begin{aligned}
P^{+}\left(t_{1}+\Delta t\right) & =\int_{t_{1}}^{t_{1}+\Delta t} p^{+}(t) d t \\
& =e^{-R\left(t_{1}-t_{0}\right)}\left(1-e^{-R \Delta t}\right) \\
& \approx e^{-R\left(t_{1}-t_{0}\right)} R \Delta t \quad \text { for } \Delta t \ll R,
\end{aligned}
$$

and the probability $P^{-}\left(t_{1}-\Delta t\right)$ to obtain the first uncorrelated event at time $t_{1} \in\left[-\infty, t_{0}\right]$ in a time bin $\Delta t$,

$$
\begin{aligned}
P^{-}\left(t_{1}-\Delta t\right) & =\int_{t_{1}-\Delta t}^{t_{1}} p^{-}(t) d t \\
& =e^{R\left(t_{1}-t_{0}\right)}\left(1-e^{-R \Delta t}\right) \\
& \approx e^{R\left(t_{1}-t_{0}\right)} R \Delta t \quad \text { for } \Delta t \ll R .
\end{aligned}
$$

The probabilities $P^{+}$and $P^{-}$are symmetric under time reversal about $t_{0}$.

If the TOF gate is opened by the start detector for $N$ times during a measurement, the uncorrelated background distribution $B_{\text {uncorr }}\left(t_{1}\right)$ for obtaining the first stop in the interval $\left[t_{1}, t_{1}+\Delta t\right]$ is calculated to be

$$
B_{\text {uncorr }}\left(t_{1}\right)=N P^{+}\left(t_{1}+\Delta t\right) \approx N e^{-R\left(t_{1}-t_{0}\right)} R \Delta t .
$$

Thus the shape of the uncorrelated background is exponential with the slope determined by the stop rate $R$ if the detector rates are used without any additional conditions in the trigger electronics.

In this experiment the stop rate for the TOF measurement is given by the $\mu_{\text {in }}$ rate. A $\mu_{\text {in }}$ is defined by the trigger logic to be not preceded by another $S_{\mu}$ hit within at least the duration of the pile-up gate length $\Delta_{\text {pu }}=2 \mu \mathrm{s}$. The $\mu_{\text {in }}$ rate is calculated using the $S_{\mu}$ rate and substituting $R$ by $S_{\mu}$ in Eq. (B4), to be

$$
\mu_{\mathrm{in}}=S_{\mu}\left[1-P^{-}\left(t_{0}-\Delta_{\mathrm{pu}}\right)\right]=S_{\mu} e^{-\Delta_{p u} S_{\mu}},
$$

where $\left[1-P^{-}\left(t_{0}-\Delta_{\mathrm{pu}}\right)\right]$ is the probability that there was no $S_{\mu}$ hit present in the past for at least $\Delta_{\mathrm{pu}}=2 \mu \mathrm{s}$. The measured $\mu_{\text {in }}$ rate of $1.7 \times 10^{5} / \mathrm{s}$ agrees with the value obtained from Eq. (B6) using the experimental $S_{\mu}$ rate of $3.6 \times 10^{5} / \mathrm{s}$ and the pile-up gate length of $2 \mu \mathrm{s}$, which is adjusted to the length of the TOF gate $\Delta_{\mathrm{TOF}}=\Delta_{\mathrm{pu}}$.

Now, we will show that the shape of the uncorrelated background in the TOF spectrum using the $\mu_{\text {in }}$ signal as the stop is flat instead of exponential. However, the final shape of the background is determined by taking into account the $S_{\mu}$ reset probability, which is derived below. The probability density $f_{\mu_{\text {in }}}\left(t_{1}\right)$ for a $\mu_{\text {in }}$ hit at time $t_{1}$ is obtained by weighting the probability density $f^{+}\left(t_{1}\right)$ for a $S_{\mu}$ at $t_{1}$ with the probability that the last $S_{\mu}$ was present in the time interval $\left[-\infty, t_{1}-\Delta_{\mathrm{pu}}\right]$ : 


$$
\begin{aligned}
& f_{\mu_{\mathrm{in}}}\left(t_{1}\right)=f^{+}\left(t_{1}\right) \int_{-\infty}^{t_{1}-\Delta_{\mathrm{pu}}} S_{\mu} e^{S_{\mu}\left(t-t_{0}\right)} d t \\
& =S_{\mu} e^{-S_{\mu} \Delta_{\mathrm{pu}}} \equiv \mu_{i n} \quad\left(\text { for } t_{1} \in\left[t_{0}, t_{0}+\Delta_{\mathrm{pu}}\right]\right) \text {. }
\end{aligned}
$$

Thus, $f_{\mu_{\text {in }}}\left(t_{1}\right)$ is flat and does not depend on the time $t_{1}$, for $t_{1} \in\left[t_{0}, t_{0}+\Delta_{\mathrm{pu}}\right]$. If the TOF gate $\Delta_{\mathrm{TOF}}$ is larger than the pile-up gate $\Delta_{\mathrm{pu}}, f_{\mu_{\mathrm{in}}}\left(t_{1}\right)=f^{+}\left(t_{1}\right)$ is exponential again for $t_{1}>\left(t_{0}+\Delta_{\mathrm{pu}}\right)$. It follows from Eq. (B7) for the uncorrelated background that

$$
B_{\text {uncorr }}\left(t_{1}\right)=N \int_{t_{1}}^{t_{1}+\Delta t} f_{\mu_{\text {in }}} d t=N \mu_{\text {in }} \Delta t
$$

for $t_{1} \in\left[t_{0}, t_{0}+\Delta_{\mathrm{pu}}\right]$, a time bin $\Delta t \leqslant \Delta_{\mathrm{pu}}$, and $N$ the number of gate openings during a measurement.

However, the $S_{\mu}$ reset probability due to pile-up modifies the shape of the background spectrum. The trigger electronics is reset if a second $S_{\mu}$ hit is detected after the detection of a $\mu_{\text {in }}$ signal while the TOF gate $\Delta_{\text {TOF }}$ is still active (see Fig. 2 ). The reset probability $r\left(t_{1}\right)$ within $\Delta_{\mathrm{TOF}}$, that an event at time $t_{1} \in\left[t_{0}, t_{0}+\Delta_{\mathrm{TOF}}\right]$ is followed by a reset in $\left[t_{1}, t_{0}\right.$ $\left.+\Delta_{\mathrm{TOF}}\right]$, is not a constant but depends on $t_{1}$ :

$$
r\left(t_{1}\right)=1-e^{-S_{\mu}\left[\Delta_{\mathrm{TOF}^{-}}-\left(t_{1}-t_{0}\right)\right]}
$$

where $t_{0}$ denotes again the start time of the TOF gate. Equation (B9) is derived by integrating $\int_{t_{1}}^{t_{0}+\Delta_{\mathrm{TOF}}} p^{-}(t) d t$, where in $p^{-}(t)$ the time zero $t_{0}$ is substituted by $\left(t_{0}+\Delta_{\mathrm{TOF}}\right)$. For $t_{1}=t_{0}$, the reset probability is at maximum whereas it becomes zero when $t_{1}$ reaches the end of the $\Delta_{\mathrm{TOF}}$ window. Thus the background distribution $B_{\text {uncorr }}\left(t_{1}\right)$ in Eq. (B8) must be corrected for the probability $\left[1-r\left(t_{1}\right)\right]$ of having no reset. This yields finally

$$
B_{\text {uncorr }}\left(t_{1}\right)=N \mu_{\text {in }} \Delta t e^{-S_{\mu}\left(\Delta_{\mathrm{TOF}}+t_{0}\right)} e^{S_{\mu} t_{1}},
$$

which again has an exponential slope, but now with the sign changed compared with Eq. (B5).

The threefold-correlated background due to decay $e^{+}$can initially be written as

$$
B_{\text {corr }}^{3 \text {-fold }}\left(t_{1}\right) \propto e^{-\left(t_{\mu}-t_{1}\right) \Gamma_{\mu}} \quad \text { for } t_{1} \leqslant t_{\mu},
$$

where $\Gamma_{\mu}=1 / \tau_{\mu}$ is the decay rate of the muon and $t_{\mu}$ denotes the time zero for $\mu^{+}$in the TOF spectrum, which is defined as the time when the $\mu^{+}$reaches the target. Again, this background has to be corrected for $\left[1-r\left(t_{1}\right)\right]$ yielding

$$
B_{\text {corr }}^{3 \text {-fold }}\left(t_{1}\right) \propto e^{-\left[S_{\mu}\left(\Delta_{\mathrm{TOF}}+t_{0}\right)+\Gamma_{\mu} t_{\mu}\right]} e^{\left(S_{\mu}+\Gamma_{\mu}\right) t_{1}} .
$$

For $t_{1}>t_{\mu}$, only uncorrelated background is possible with a shape $\propto e^{S_{\mu} t_{1}}$.

The twofold-correlated background (MCP1 and $\mathrm{S}_{e i}$ correlated and $\mu_{\text {in }}$ uncorrelated) due to decay $e^{+}$follows Eq. (B10), with $N$ substituted by an unknown $N^{\prime}$. Finally, the total background $B(t)$ in the reverse timing spectrum is a sum of Eqs. (B10) and (B12):

$$
\begin{array}{r}
B(t)=a_{\text {uncorr }} e^{-S_{\mu}\left(\Delta_{\mathrm{TOF}}+t_{0}\right)} e^{S_{\mu} t} \\
+a_{\mathrm{corr}} e^{-\left[S_{\mu}\left(\Delta_{\mathrm{TOF}}+t_{0}\right)+\Gamma_{\mu} t_{\mu}\right]} e^{\left(S_{\mu}+\Gamma_{\mu}\right) t} \\
\quad \text { for } t \leqslant t_{\mu},
\end{array}
$$

where $a_{\text {uncorr }}$ and $a_{\text {corr }}$ are fit parameters, and $t_{1}$ is substituted by $t$. For $t>t_{\mu}, B(t)$ reduces to the first term in Eq. (B13).

In order to calculate $B\left(t^{\prime}\right)$ for the forward timing spectra, which are shown in Figs. 3(a) and 4, a variable substitution $t=-t^{\prime}+t_{\mu}$ is performed to yield

$$
\begin{gathered}
B\left(t^{\prime}\right)=e^{-S_{\mu}\left(\Delta_{\mathrm{TOF}}+t_{0}-t_{\mu}\right)} \\
\times\left(a_{\text {uncorr }} e^{-S_{\mu} t^{\prime}}+a_{\text {corr }} e^{-\left(S_{\mu}+\Gamma_{\mu}\right) t^{\prime}}\right) \\
\text { for } t^{\prime}>0 .
\end{gathered}
$$

For $t^{\prime}<0$, only the uncorrelated term contributes to the background. Also, the correlated term is removed after applying the cuts described in Sec. III. In Fig. 4(b), only the first term of Eq. (B14) is fitted, with $a_{\text {uncorr }}$ as the fit parameter.

The integral rate of uncorrelated background $B_{\text {uncorr }}$ is estimated using the measured rate of TOF coincidences ( $\left.\mu_{\text {in }} \cdot \mathrm{MCP} 1\right)$ and the rate $S_{e}$ of the $\mathrm{S}_{e i}$ detectors. Given a ( $\left.\mu_{\text {in }} \cdot \mathrm{MCP} 1\right)$ coincidence, the probability of having at least one event in the scintillator pairs is $P^{+}\left(t_{0}+10 \mu \mathrm{s}\right) \approx S_{e}$ $\times 10 \mu$ s according to Eq. (B3), where $S_{e}$ substitutes $R$, the $10-\mu \mathrm{s}$ master gate length substitutes $\Delta t$, and $S_{e} \times 10 \mu s$ $\ll 1$ is used. With the rates in the Mu TOF measurements of $\left(\mu_{\text {in }} \cdot \mathrm{MCP} 1\right) \approx 54 / \mathrm{s}$ and $S_{e} \approx 100 / \mathrm{s}$, it follows that

$$
B_{\text {uncorr }}=\left(\mu_{\text {in }} \cdot \mathrm{MCP} 1\right) S_{e} \times 10 \mu \mathrm{s} \approx 0.054 / \mathrm{s} \text {, }
$$

where the $\left(\mu_{\text {in }} \cdot \mathrm{MCP} 1\right)$ rate is the accepted coincidence rate after pile-up rejection.

The integral background rate $B_{\text {target }}$ of decay $e^{+}$originating at the target is estimated as follows. Taking into account the spatially anisotropic $e^{+}$distribution in the $\mu^{+}$decay [30], a fraction of $\Omega_{e+}=3 \times 10^{-4}$ hits the MCP1 detector. The GEANT simulation shows that there is a probability $\epsilon_{e+}^{\text {scatt }} \approx 0.02$ of scattering a $e^{+}$in MCP1 toward the direction of the scintillator pairs. The decay $e^{+}$are relativistic, so the detection efficiency in MCP1 is expected to be $\epsilon_{\mathrm{MCP} 1}^{e+} \leqslant 0.1$ [31]. Now, the background rate $B_{\text {target }}$ can be estimated to be

$$
B_{\text {target }}=\mu_{\text {in }}^{\text {acc }} \epsilon_{\text {stop }} \Omega_{e+} \epsilon_{e+}^{\text {scatt }} \epsilon_{\mathrm{MCP} 1}^{e+} \approx 0.05 / \mathrm{s},
$$

where $\mu_{\text {in }}^{\text {acc }}=10^{5} / \mathrm{s}$ denotes the accepted beam rate, and $\epsilon_{\text {stop }}$ $=0.87$ is the fraction of the beam stopping in the target region. This background is negligible for the $N_{\text {tot }}(t)$ data, but contributes to a large extent to the background in the $\mathrm{Mu}$ measurement. 
The measured Mu TOF background rate of $0.15 / \mathrm{s}$ originating from the prompt peak (which is the sum of the twofold- and threefold- correlated background rates), together with the estimated $B_{\text {uncorr }}$ rate in Eq. (B15), are in agreement with the measured trigger rate (approximately equal to the background rate) of $0.2 / \mathrm{s}$. From the fit of Eq. (B14) to the Mu TOF spectra without cuts [see Fig. 4(a)], a threefold-correlated background fraction of $0.31(6)$ of the total background is obtained by integrating the dotted function in Fig. 4(a). This can be checked by considering that a fraction of $0.5 \times 0.15 / \mathrm{s}=0.075 / \mathrm{s}$ of the "prompt peak" background rate is expected to cause the threefold-correlated background, because within the 1.6- $\mu$ s TOF gate length between the $\mu^{+}$time zero and the end of the gate, (1 $\left.-e^{-1.6 \mu \mathrm{s} / \tau} \mu\right)=50 \%$ of the $\mu^{+}$decay. Thus a fraction of $0.075 / 0.2=0.375$ of the total background rate is expected from this estimate. Within the error, this is in agreement with the result obtained independently from the fit.
[1] J. A. Phillips, Phys. Rev. 97, 404 (1955).

[2] K. Allison, Rev. Mod. Phys. 30, 1137 (1958).

[3] H. Tawara and A. Russek, Rev. Mod. Phys. 45, 178 (1974).

[4] R. Shakeshaft and L. Spruch, Rev. Mod. Phys. 51, 369 (1979).

[5] G. G. Ross and L. Leblanc, Nucl. Instrum. Methods Phys. Res. B 48, 134 (1990).

[6] For a recent theoretical review, see B. H. Bransden and M. R. C. McDowell, Charge Exchange and the Theory of Ion-Atom Collisions (Clarendon, Oxford, 1992).

[7] K. Shima, N. Kuno, M. Yamanouchi, and H. Tawara, At. Data Nucl. Data Tables 51, 173 (1992).

[8] A. Bürgi, M. Oetliker, P. Bochsler, J. Geiss, and M. A. Coplan, J. Appl. Phys. 68, 2547 (1990).

[9] See, for example, Particle Data Group, Phys. Rev. D 54, 1 (1996).

[10] P. R. Bolton, A. Badertscher, P. O. Egan, C. J. Gardner, M. Gladisch, V. W. Hughes, D. C. Lu, M. Ritter, P. A. Souder, J. Vetter, G. zu Putlitz, M. Eckhause, and J. Kane, Phys. Rev. Lett. 47, 1441 (1981).

[11] Y. Kuang, K. P. Arnold, F. Chmely, M. Eckhause, V. W. Hughes, J. R. Kane, S. Kettell, D. H. Kim, K. Kumar, D. C. Lu, B. E. Matthias, B. Ni, H. Orth, G. zu Putlitz, H. R. Schaefer, P. A. Souder, and K. Woodle, Phys. Rev. A 39, 6109 (1989).

[12] H. E. Ahn, Ph.D. thesis, Yale University, 1992 (unpublished).

[13] A. Hofer, E. Morenzoni, M. Birke, H. Glückler, J. Litterst, M. Meyberg, Ch. Niedermayer, T. Prokscha, G. Schatz, and T. Wutzke (unpublished).

[14] H. U. Martin, in Quantum Electrodynamics, edited by T. Kinoshita (World Scientific, Singapore 1990), p. 92; T. Kinoshita and W. J. Marciano, ibid., p. 419.

[15] See, for example, The Future of Muon Physics, edited by K. Jungmann, V. W. Hughes, and G. zu Putlitz (Springer, Heidelberg 1992).

[16] R. Abela, J. Bagaturia, W. Bertl, R. Engfer, B. Fischer von Weikersthal, A. Groß mann, V. W. Hughes, K. Jungmann, D. Kampmann, V. Karpuchin, I. Kisel, A. Klaas, S. Korenchenko, N. Kuchinsky, A. Leuschner, B. E. Matthias, R. Menz, V. Meyer, D. Mzavia, G. Otter, T. Prokscha, H. S. Pruys, G. zu Putlitz, W. Reichart, I. Reinhard, D. Renker, T. Sakhelashvilli, P. V. Schmidt, R. Seeliger, H. K. Walter, L. Willmann, and L. Zhang, Phys. Rev. Lett. 77, 1950 (1996).

[17] A. Schenck, Muon Spin Rotation Spectroscopy (Hilger, Bristol, 1985).

[18] D. G. Fleming, R. J. Mikula, and D. M. Garner, Phys. Rev. A 26, 2527 (1982).
[19] M. Senba, J. Phys. B 21, 3093 (1988); 22, 2027 (1989); 23, 1545 (1990).

[20] V. Storchak, J. H. Brewer, and G. D. Morris, Phys. Lett. A 193, 199 (1994).

[21] E. Krasnoperov, E. Meilikhov, R. Abela, D. Herlach, E. Morenzoni, F. N. Gygax, A. Schenck, and D. Eschenko, Phys. Rev. Lett. 69, 1560 (1992).

[22] V. Storchak, J. H. Brewer, W. N. Hardy, S. R. Kreitzman, and G. D. Morris, Hyperfine Interact. 85, 109 (1994).

[23] D. R. Harshman, A. P. Mills Jr., J. L. Beveridge, K. R. Kendall, G. D. Morris, M. Senba, J. B. Warren, A. S. Rupaal, and J. H. Turner, Phys. Rev. B 36, 8850 (1987).

[24] E. Morenzoni, F. Kottmann, D. Maden, B. E. Matthias, M. Meyberg, Th. Prokscha, Th. Wutzke, and U. Zimmermann, Phys. Rev. Lett. 72, 2793 (1994).

[25] E. Morenzoni, M. Birke, H. Glückler, A. Hofer, J. Litterst, M. Meyberg, Ch. Niedermayer, Th. Prokscha, G. Schatz, and Th. Wutzke, Hyperfine Interact. 106, 229 (1997).

[26] E. Morenzoni, Th. Prokscha, A. Hofer, B. E. Matthias, M. Meyberg, Th. Wutzke, H. Glückler, M. Birke, J. Litterst, Ch. Niedermayer, and G. Schatz, J. Appl. Phys. 81, 3340 (1997).

[27] Y. Nakai, T. Shirai, T. Tabata, and R. Ito, Nucl. Data Tables 37, 69 (1987).

[28] M. C. Cross, Phys. Rev. B 15, 602 (1977).

[29] A. E. Pifer, T. Bowen, and K. R. Kendall, Nucl. Instrum. Methods 135, 39 (1976).

[30] See, for example, F. Scheck, Phys. Rep. 44, 188 (1978).

[31] D. de Bruin, P. van Deenen, D. Dijkkamp, H. Holsboer, and C. van Oven, FOM-Instituut voor Atoom-en Molecuulfysica, Technical Report No. AMOLF 89/82, 1982 (unpublished).

[32] CN division/CERN, GEANT_Detector Description and Simulation Tool, Geneva, 1993 (unpublished).

[33] K. H. Berkner, I. Bornstein, R. V. Pyle, and J. W. Stearns, Phys. Rev. A 6, 278 (1972).

[34] G. Molière, Z. Naturforschung 3A, 78 (1948).

[35] H. H. Anderson and J. F. Ziegler, Hydrogen: Stopping Powers and Ranges in all Elements (Pergamon, New York 1977).

[36] See, for example, B. Rossi, High Energy Particles (PrenticeHall, New York, 1965), p. 37.

[37] P. M. Echenique, F. Flores, and R. H. Ritchie, Nucl. Instrum. Methods Phys. Res. B 33, 91 (1988).

[38] M. Gonin, R. Kallenbach, and P. Bochsler, Rev. Sci. Instrum. 65, 648 (1994).

[39] R. Kallenbach, M. Gonin, A. Bürgi, and P. Bochsler, Nucl. Instrum. Methods Phys. Res. B 83, 68 (1993).

[40] T. Ohdaira, H. Matsushita, A. Takashima, N. Imanishi, and F. Fukuzawa, Phys. Rev. A 47, 236 (1993). 
[41] B. H. Bransden and C. Forster, J. Phys. B 23, 115 (1990).

[42] See, Charge Exchange and the Theory of Ion-Atom Collisions (Ref. [6]), p. 184.

[43] See, Charge Exchange and the Theory of Ion-Atom Collisions (Ref. [6]), p. 121.

[44] J. R. Kempton, M. Senba, D. J. Arsenau, A. C. Gonzalez, J. J. Pan, A. Tempelmann, D. M. Garner, and D. G. Fleming, Hyperfine Interact. 65, 801 (1990).
[45] J. R. Kempton, M. Senba, D. J. Arsenau, A. C. Gonzalez, D. M. Garner, J. J. Pan, D. G. Fleming, P. W. Percival, J. C. Brodovitch, and K. L. Siu, J. Chem. Phys. 94, 1046 (1991).

[46] R. F. Kiefl, J. B. Warren, G. M. Marshall, C. J. Oram, and C. W. Clawson, J. Chem. Phys. 74, 308 (1981).

[47] W. T. Eadie, D. Drijard, F. E. James, M. Roos, and B. Sadoulet, Statistical Methods in Experimental Physics (NorthHolland, Amsterdam, 1971). 